\title{
PRONÓSTICO DEL CRECIMIENTO TRIMESTRAL DE COSTA RICA MEDIANTE MODELOS DE FRECUENCIA MIXTA ${ }^{1}$
}

\author{
Adolfo Rodríguez Vargas ${ }^{2}$ \\ Recibido: 01/08/2014 Aprobado: 19/09/2014 \\ RESUMEN
}

\begin{abstract}
Se evalúa la utilidad de modelos de frecuencia mixta para pronosticar la tasa de crecimiento trimestral del PIB real de Costa Rica: se estiman modelos bridge y MiDaS con diferentes longitudes de rezago usando información del IMAE y se calculan pronósticos (horizontes de 0-4 trimestres) que se comparan entre sí, con los de modelos ARIMA y con combinaciones de pronósticos. Combinar los pronósticos con mejor ajuste resulta útil especialmente para proyectar en tiempo real, mientras que los MiDaS muestran el mejor desempeño general: al incrementarse el horizonte su precisión disminuye levemente, su porcentaje de acierto de cambios en la tasa de variación del producto permanece estable y varios de ellos son insesgados. Los pronósticos de MiDaS simples con 9 y 12 rezagos resultaron insesgados para todos los horizontes y conjuntos de información evaluados, y son los que mostraron más diferencias significativas en capacidad de pronóstico con todos los demás modelos.
\end{abstract}

PALABRAS CLAVE: DATOS DE FRECUENCIA MIXTA, MODELOS MIDAS, MODELOS BRIDGE, PRONÓSTICO EN TIEMPO REAL.

\begin{abstract}
We assess the utility of mixed-frequency models to forecast the quarterly growth rate of Costa Rican real GDP: we estimate bridge and MiDaS models with several lag lengths using information of the IMAE and compute forecasts (horizons of 0-4 quarters) which are compared between themselves, with those of ARIMA models and with those resulting from forecast combinations. Combining the most accurate forecasts is most useful when forecasting in real time, whereas MiDaS forecasts are the best-performing overall: as the forecasting horizon increases, their precisionis affected relatively little; their success rates in predicting the direction of changes in the growth rate are stable, and several forecasts remain unbiased. In particular, forecasts computed from simple MiDaS with 9 and 12 lags are unbiased at all horizons and information sets assessed, and show the highest number of significant differences in forecasting ability in comparison with all other models.
\end{abstract}

KEY WORDS: MIXED-FREQUENCY DATA, MIDAS MODELS, BRIDGE MODELS, NOWCASTING.

1 Las ideas expresadas en estos documentos son de los autores y no necesariamente representan las del Banco Central de Costa Rica. 


\section{INTRODUCCIÓN}

Para formular políticas pertinentes, las autoridades económicas requieren evaluar en tiempo real el estado de la economía, así como proyectar la evolución en el mediano plazo de variables clave, utilizando información usualmente incompleta para el periodo de referencia. En particular, para un banco central es de especial interés contar de manera oportuna con estimaciones del crecimiento del producto pues además de ser uno de los indicadores de actividad económica más cercanamente seguidos por los agentes económicos, le ayudaría a anticipar la formación de presiones inflacionarias. Sin embargo, los datos del Producto Interno Bruto (PIB) y de otros agregados trimestrales por lo general tienen un rezago de publicación considerable. Aunado a esto, gran parte de los modelos econométricos de bancos centrales pronostican en el momento $t$ la evolución del producto trimestral en $t+k$, usando variables que usualmente tienen la misma periodicidad que el producto $y$ cuyos datos también se tienen disponibles con rezago.

Para solventar esa limitación se han desarrollado modelos de proyección que aprovechan la información de indicadores de mayor frecuencia disponibles más oportunamente. Este tipo de modelos se conocen como de frecuencia mixta, y posibilitan, por ejemplo, obtener pronósticos del crecimiento del PIB trimestral a partir de información de un indicador mensual de actividad; del precio del petróleo a fin de mes a partir de indicadores financieros y de precios con frecuencia semanal; o de la volatilidad mensual del retorno de un activo usando información financiera diaria.

Esta clase de métodos de pronóstico incluye los modelos bridge, los modelos de "Mixed Data Sampling" (MiDaS), así como métodos de estado-espacio como los VAR de frecuencia mixta y los modelos de factores. Las dos primeras metodologías son de uso especialmente extendido en departamentos de investigación y de estadística de bancos centrales ${ }^{3}$. En general, todos estos métodos han mostrado ganancias significativas en la capacidad de pronóstico de varias variables económicas en aplicaciones realizadas por académicos y por investigadores de bancos centrales ${ }^{4}$. Esta mayor capacidad de pronóstico permite mejorar los pronósticos de otros modelos en los que sean insumos variables trimestrales con rezagos de publicación.

El objetivo de este documento es evaluar la utilidad de modelos de frecuencia mixta para pronosticar la tasa de crecimiento trimestral del PIB real de Costa Rica. Para ese fin se estiman modelos bridge y modelos MiDaS con diferentes longitudes de rezago a partir de información de un indicador mensual de actividad económica, y sus pronósticos se comparan entre sí y con los de un modelo ARIMA. Se utiliza como insumo solamente datos del Índice Mensual de Actividad Económica (IMAE) porque es el indicador de actividad que está disponible más rápidamente ${ }^{5}$. Utilizar información de un único indicador tiene la ventaja de agilizar las proyecciones del producto en comparación con modelos con más insumos.

El resto de este documento se estructura como sigue: la siguiente sección describe brevemente los modelos bridge y MiDaS y lista literatura relevante sobre ellos, en la sección 3 se exponen los detalles relacionados con los datos y la estimación, en la sección 4 se presentan los pronósticos y los resultados de su evaluación, y finalmente la sección 5 contiene las principales conclusiones del estudio.Modelos bridge y MiDaS

Los modelos de regresión tradicionales, en los cuales se basa una parte considerable de los pronósticos de bancos centrales, se realizan con datos que poseen la misma frecuencia. Los métodos

$3 \quad$ Véase, Klein y Sojo (1989), Trehan (1992) e Ingenito y Trehan (1996).

4 Véase por ejemplo Bulligan, Marcellino y Venditti (2012); Antipa, Barhoumi, Brunhes-Lesange y Darné (2012) y Golinelli y Parigi (2007).

$5 \quad$ Los datos de empleo no están disponibles con frecuencia mensual. A partir del 3er trimestre del 2010 el Instituto Nacional de Estadística y Censos (INEC) publica datos trimestrales derivados de la Encuesta Continua de Empleo, con un trimestre de rezago. 
que se describen en esta sección permiten realizar pronósticos utilizando información de variables medidas con una frecuencia más alta que la de la variable a proyectar. Por ejemplo, permiten proyectar variables trimestrales con base en variables mensuales o variables mensuales a partir de información diaria. Dado el objetivo de este documento, la breve exposición de las metodologías que se realiza en esta sección toma como referencia la tasa de variación trimestral del PIB y variables de frecuencia mensual.

\section{Modelos bridge}

Los modelos bridge o de puente ${ }^{6}$ se utilizan para relacionar indicadores mensuales de actividad económica $Z_{\mathrm{i}}$, con la variación del PIB del trimestre contemporáneo, $\mathrm{Y}_{\mathrm{t}}$. La idea es aprovechar la información mensual más inmediatamente disponible en la proyección de la variable trimestral. Para esto, a partir de los indicadores mensuales $Z_{\mathrm{i}}$ se generan variables explicativas trimestrales $\mathrm{X}_{\mathrm{i}}$ que se incluirán en la modelación de la ecuación bridge.

El método fue desarrollado por Klein y Sojo (1989) para la economía estadounidense, y algunos ejemplos tempranos de su aplicación son Trehan (1992) e Ingenito y Trehan (1996) para los Estados Unidos de América y Parigi y Schlitzer (1995) para Italia. Desde entonces el método se ha utilizado con frecuencia para la proyección del crecimiento trimestral del PIB, particularmente en la zona Euro. Algunos estudios más recientes de aplicación son Bulligan, Marcellino y Venditti (2012) para la zona Euro; Antipa, Barhoumi, Brunhes-Lesange y Darné (2012) para Alemania; Cobb et al. (2011) para Chile; Barhoumi, Brunhes-Lesange, Darné, Ferrara, Pluyaud y Rouvreaul (2008) y Barhoumi, Darné, Ferrara y Pluyaud (2012) para Francia; y Golinelli y Parigi (2007) para varios países industrializados.

Un modelo bridge de proyección trimestral es un modelo autorregresivo de rezagos distribuidos:

$$
Y_{t}=\alpha+\sum_{i=1}^{p} \beta_{i} Y_{t-i}+\sum_{j=1}^{k} \sum_{i=1}^{q} \gamma_{j, i} X_{j, t-i}+\varepsilon_{t}
$$

en el que $p$ es el número de términos autorregresivos, $k$ es el número de variables explicativas y $q$ es el número de rezagos de dichas variables explicativas.

La estimación de este tipo de modelos usualmente comprende dos pasos:

- Selección de un conjunto de variables mensuales que puedan resultar de utilidad para predecir la variación del PIB. La selección considera relaciones teóricas entre las variables, así como evidencia empírica de correlación.

- Selección de la parametrización de (1).

Para determinar la especificación de la ecuación bridge es común el uso del procedimiento de estimación general a específico propuesto por David Hendry. El uso de esta metodología es estándar en la literatura sobre pronóstico en tiempo real, por cuanto reduce la subjetividad en la selección de la especificación y facilita la reestimación expedita de los modelos ante cambios en los datos ${ }^{7}$. Se parte de un modelo general irrestricto (MGI), que corresponde al modelo más general que se consi-

$\overline{6} \quad$ En adelante se utilizará el término en inglés por brevedad y por ser el de uso más difundido.

$7 \quad$ Algunos estudios que utilizan metodología general a específico para la selección de ecuaciones bridge son Bulligan et al (2012); Antipa et al (2012); Cobb et al. (2011); Barhoum et al (2008) y Barhoumi et al (2012); y Golinelli y Parigi (2007). 
dera razonable para el proceso generador de datos (PGD), y que incluye todas las variables potencialmente relevantes, así como el número máximo de rezagos con los que entran. A partir de este punto se inicia un proceso sistematizado de pruebas estadísticas y reestimación para reducir el PGD hasta llegar a una especificación final satisfactoria, que se considera la aproximación más exacta del PGD desconocido. Es claro entonces que un modelo bridge no necesariamente debe tener una interpretación teórica.

Hoover y Pérez (1999) fueron los primeros en proponer un algoritmo para la aplicación automatizada del método general a específico. Krolzig y Hendry (2001) extendieron y mejoraron ese algoritmo, $y$ se convirtieron en una de las referencias más usadas en la estimación de modelos bridge. Esa es la metodología utilizada para la estimación de las ecuaciones bridge utilizadas en este estudio $^{8}$. Las etapas del método se pueden resumir de la siguiente forma ${ }^{9}$ :

1. Formulación, estimación y prueba del MGI.

2. Eliminación de variables insignificantes del MGI mediante un proceso previo a la búsqueda (presearch lag reduction).

3. Búsquedas de especificación en varias vías para la reducción del MGI, iniciando cada vía en cada eliminación inicial factible (multi-path reduction searches). Las reducciones que pasan un diagnóstico de validez para asegurar la congruencia a los datos se clasifican como selecciones terminales. El proceso implica identificar todas las selecciones terminales no dominadas por otras, formar nuevos MGI con la unión de estas selecciones terminales y repetir el proceso de reducción hasta encontrar un modelo final (o un grupo de modelos finales de entre los cuales se escoge uno).

4. Evaluación posterior a la búsqueda utilizando muestras traslapadas para verificar la confiabilidad de la selección.

Las pruebas de especificación usadas para el diagnóstico en este proceso automatizado son:

- Pruebas F de significancia para grupos de coeficientes.

- Pruebas de normalidad (Jarque y Bera, 1980; Doornik y Hansen, 1994).

- Pruebas de heterocedasticidad (White, 1980; Nicholls y Pagan, 1983; Hendry y Doornik, 1996).

- Prueba de multiplicador de Lagrange de Godfrey (1978) para correlación serial hasta 5 rezagos.

- Prueba portmanteau para correlación serial (Box y Pierce, 1970).

- Pruebas de Chow para estabilidad de coeficientes (Chow, 1960; Hendry, 1979).

Una vez definida la parametrización de la ecuación (1), el modelo se reestima cada vez que hay información nueva para las variables explicativas y se utiliza para realizar pronósticos de la variables de frecuencia más baja. Si la proyección se realiza cuando se tiene disponible información para el primero o segundo mes del trimestre, es necesario completar la información de los meses faltantes de cada $Z_{\mathrm{it}}$. Esto se suele realizar mediante modelos auxiliares para pronosticar cada indicador. Por ejemplo, si se tiene disponible información para el primer mes del trimestre, mediante un modelo auxiliar, usualmente una ARIMA, se proyecta los meses segundo y tercero. Una vez que se cuenta con todos los datos mensuales del trimestre, tanto observados como pronosticados, se construyen las variables $\mathrm{X}_{\mathrm{it}}$, por lo general tomando el promedio de las observaciones mensuales $Z_{\mathrm{it}}$ . En el caso de contar con indicadores mensuales con publicación anterior a la del PIB trimestral,

8 Doornik y Hendry (2007) desarrollaron un algoritmo de tercera generación, implementado en software no disponible para el autor al momento de la realización de este estudio.

$9 \quad$ Ver Krolzig y Hendry (2001) y Krolzig y Hendry (2005) para los detalles completos. 
cuando se tenga información del tercer mes del trimestre no sería necesario utilizar los modelos auxiliares para completar los datos del trimestre y se podría realizar nowcasting.

\section{Modelos MiDaS}

A diferencia de un modelo bridge, en el que la información mensual se agrega a una frecuencia trimestral antes de estimar el modelo de regresión para pronóstico, en los modelos de regresión Mixed Data Sampling (MiDaS) se utiliza un conjunto de información más amplio, en el que se combina datos de diferente frecuencia mediante un esquema de ponderación parsimonioso. Siguiendo a Ghysels, Santa-Clara y Balkanov (2004), un modelo MiDaS puede representarse como sigue.

Supóngase que la variable $Y_{t}$ tiene una frecuencia (semanal, mensual, trimestral, anual...) y llámese a esa frecuencia el intervalo de referencia. Ahora, supóngase que la variable $X_{i}^{(m)}$ tiene una frecuencia $m$ veces más rápida, de modo que si $Y_{t}$ tiene frecuencia trimestral, $m=3$ implica que $X_{i}^{(3)}$ es una variable mensual. La regresión lineal simple MIDAS está dada por

$$
Y_{t}=\beta_{0}+\beta_{1} B\left(L^{1 / m}\right) X_{t}^{(m)}+\varepsilon_{t}^{(m)}
$$

donde $B\left(L^{1 / m}\right)$ es un polinomio de longitud $j^{\text {max }}$ del operador de rezagos $\left(L^{1 / m}\right)$ :

$$
B\left(L^{1 / m}\right)=\sum_{j=0}^{j^{\max }} \omega_{j}\left(\theta^{(m)}\right) L^{j / m}
$$

Así $L^{j / m} X_{t}^{(m)}=X_{t-j / m}^{(m)}$. En el ejemplo que se presenta la regresión MiDaS es una proyección de la variable trimestral $Y_{t}$ sobre datos mensuales $X_{t}^{(m)}$ usando hasta $j^{\text {max }}$ rezagos.

El número de parámetros en el polinomio depende de $m$ y del número de rezagos incluidos. Por ejemplo, un polinomio con 4 rezagos en un caso de estimación de variable trimestral con datos mensuales implicaría la necesidad de estimar 12 parámetros. Como puede verse, el conjunto de información más amplio que usa el enfoque MiDaS tiene un costo en términos de proliferación de parámetros. Por ello, se han sugerido varias parametrizaciones para el polinomio de rezagos, que se describen brevemente a continuación. Para mayor detalle consultar Ghysels, Sinko y Valkanov (2006) y Ghysels (2014).

1. Polinomio MiDaS no restringido (U-MiDaS): los coeficientes del polinomio $B\left(L^{1 / m}\right)$ se estiman sin restricción. Este enfoque ha mostrado funcionar bien para especificaciones con bajo $m_{s}$ como el caso de modelos con esquema de muestreo mensual/trimestral.

2. Función de densidad en probabilidad beta normalizada: se pueden calcular versiones restringida $y$ no restringida, $y$ con rezago final cero $y$ diferente de cero.

3. Polinomio de rezagos de Almon exponencial normalizado: para $\mathrm{N}$ rezagos, los pesos $\omega_{\mathrm{i}}$, tanto restringidos $(r)$ como no restringidos $(u)$ deben sumar 1 y son función de los parámetros a esti$\operatorname{mar} \theta^{(m)}=\left(\theta_{1}, \theta_{2}\right)$ :

$$
\begin{aligned}
& \omega_{i}^{u}=\omega_{i}\left(\theta_{1}, \theta_{2}\right)=\frac{e^{\theta_{1} i+\theta_{2} i^{2}}}{\sum_{i=1}^{N} e^{\theta_{1} i+\theta_{2} i^{2}}} \\
& \omega_{i}^{r}=\omega_{i}\left(\theta_{1}, 0\right)
\end{aligned}
$$


4. Polinomio de rezagos de Almon de orden $P$, no normalizado: se estima conjuntamente $\beta_{1} B\left(L^{1 / m}\right)$, de manera que:

$$
\beta \omega_{i}\left(\theta_{0}, \ldots, \theta_{p}\right)=\sum_{p=0}^{P} \theta_{p} i^{p}
$$

Los pesos pueden calcularse mediante mínimos cuadrados ordinarios a partir de una transformación de los datos de alta frecuencia (ver Ghysels, 2014) y luego pueden re escalarse para obtener el coeficiente $\beta$.

5. Especificación polinomial con funciones de grada, no normalizada.

Es importante destacar que la regresión (2) no necesariamente debe ser lineal o univariada. Además, puede aumentarse para incluir variables exógenas medidas con la periodicidad más baja, así como rezagos de la variable dependiente Yi, lo que da origen a un MiDaS autorregresivo (ADL-MiDaS).

Una especificación de particular interés es el MiDaS con adelantos. Estos modelos son útiles pues permiten incorporar valores de la variable de alta frecuencia observados para el trimestre vigente, por lo que es factible realizar pronóstico en tiempo real de la variable de frecuencia más baja (ver Ghysels, 2014), un ejercicio que se realiza en el presente trabajo.

Los parámetros $\left(\beta_{0}, \beta_{1}, \theta^{(m)}\right)$ por lo general se estiman conjuntamente mediante mínimos cuadrados no lineales.

Se puede consultar sobre extensiones adicionales del modelo MiDaS en Chen y Ghysels (2011), Ghysels (2012) y Engle, Ghysels y Sohn (2013), entre otros. Algunos estudios donde se aplica modelos MiDaS para predecir variables macroeconómicas son Winkelried (2012) para Perú; Kuzin, Marcellino y Schumacher (2011) para la zona Euro; y Clements y Galvão (2006), y Armesto, Engemann y Owyang (2010) para los EE.UU.

\section{Datos y estimación de los modelos}

\section{- Datos}

En Costa Rica, los datos del PIB trimestral deben estar disponibles con un rezago de un trimestre tras el final del trimestre de referencia, mientras que el indicador de actividad mensual disponible con mayor celeridad, el IMAE, se publica con un rezago 42 días tras la finalización del mes de referencia ${ }^{10}$. Como se ilustra en la figura 1 , esto implica que:

- Es factible realizar pronóstico en tiempo real (nowcasting) del PIB trimestral en el último mes del trimestre de referencia.

- Los tres datos del IMAE de cada trimestre están disponibles aproximadamente 7 semanas antes de la publicación de la cifra oficial del PIB trimestral correspondiente, por lo que con esa antelación se podría tener pronósticos para el dato trimestral basados en información mensual completa para el trimestre.

10 El calendario actualizado de publicación de cifras del BCCR puede consultarse en la dirección electrónica: http://indicadoreseconomicos.bccr.fi.cr/indicadoreseconomicos/Documentos/NEDD/Calendario-esp.htm 
El IMAE se calcula a partir de indicadores mensuales de todas las industrias de la Clasificación Internacional Industrial Uniforme (CIIU), revisión 2. En el anexo 1 se lista los 12 índices considerados para calcular las tasas de variación usadas en la especificación general irrestricta de los modelos bridge ${ }^{11}$.

FIGURA 1

CALENDARIZACIÓN DE PUBLICACIÓN DE CIFRAS SOBRE ACTIVIDAD ECONÓMICA EN COSTA RICA

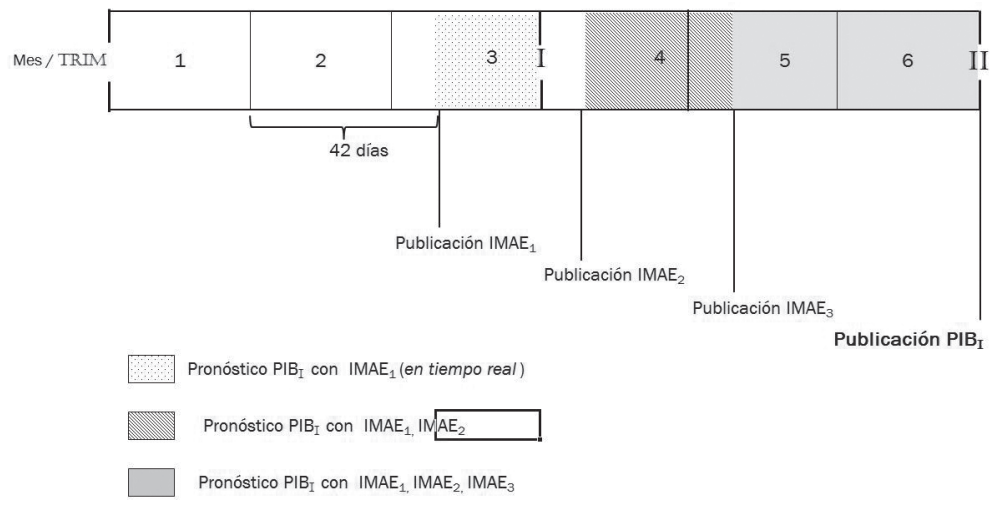

Fuente: elaboración propia.

Los datos utilizados en las estimaciones son las tasas de variación del IMAE tendencia ciclo, así como de sus componentes, con respecto a su valor tres meses atrás (vimae); y la tasa de variación trimestral del PIB real, tendencia ciclo (vpib). Se utilizó la información vigente al 14 de noviembre de 2013. Las muestras de estimación abarcan de 1992-II a 2009-II inclusive, dependiendo del horizonte de pronóstico, mientras que la muestra de evaluación común a todos los pronósticos es 2009-III - 2013-II (16 observaciones).

Mediante la prueba de Bai y Perron (2003) se determinó que no existe evidencia de cambio estructural en las series de tasas de variación del PIB y del IMAE. Además, las pruebas ADF y Phillips-Perron indican que no existe evidencia de raíz unitaria. En el anexo 2 se pueden consultar los detalles de todas las pruebas.

\section{- $\quad$ Estimación de la ecuación bridge}

La especificación general irrestricta de la que se partió en este estudio corresponde a un modelo ARDL con datos del IMAE como variables explicativas. Se realizó dos estimaciones: una que utilizó la tasa de variación trimestral del IMAE tendencia - ciclo (vimaet) y otra en la que se utilizaron las tasas de variación trimestrales de cada uno de los índices por industria que componen el IMAE, (vimaetj, $\mathrm{j}=1,2, \ldots, 12$ ). En ambos casos la tasa de variación trimestral del IMAE se obtuvo como el promedio de las tasas vimae de los meses correspondientes. Se partió de un especificaciones con 4 $y$ con 8 rezagos de la variable dependiente $y$ de las explicativas, $y$ la búsqueda de la especificación se realizó contemplando detección de valores extremos y eliminación previa de variables no relevantes. La muestra de estimación fue 1991-II a 2009-II, de forma que se dejara 4 años de datos para la muestra de evaluación de los pronósticos. La especificación escogida se muestra en el cuadro 1.

11 La metodología de cálculo del IMAE puede consultarse en BCCR (s.f). 
La selección del modelo final fue robusta a diferentes especificaciones iniciales del MGI en la estimación con las tasas de variación del IMAE agregado. Se obtuvo el mismo modelo final aun si en el MGI con 4 rezagos se incluía variables indicadoras estacionales centradas o no centradas, y se omitía el proceso de eliminación previa de variables no relevantes. Cuando se consideró 8 rezagos, con la mayoría de variantes de la especificación del MGI, se obtuvo la misma estructura de rezagos del cuadro 1. En el caso de la estimación con las tasas de variación del IMAE desagregado, la elección del modelo fue más sensible a la especificación inicial del MGI, especialmente cuando se consideró 8 rezagos. Se optó por utilizar la especificación más parsimoniosa del cuadro 1, que utiliza datos del IMAE agregado y que mostró un ajuste mejor al del resto de opciones.

\section{CUADRO 1 \\ ECUACIÓN BRIDGE ESCOGIDA \\ DATOS IMAE AGREGADO}

\begin{tabular}{ccccc}
\hline Variable & Coeficiente & Error estándar & Estadístico $\mathrm{t}$ & Valor $\mathrm{p}$ \\
\hline & & & & \\
Constante & 0,6159 & 0,0752 & 8,19 & 0,0000 \\
vimaet & 0,3629 & 0,0483 & 7,52 & 0,0000 \\
vimaet $_{\mathrm{t}-1}$ & 0,1394 & 0,0522 & 2,67 & 0,0097 \\
vimaet $_{\mathrm{t}-2}$ & 0,0642 & 0,0477 & 1,34 & 0,1837 \\
D1995IV & $-3,6820$ & 0,3654 & $-10,1$ & 0,0000 \\
D1997I & 1,0112 & 0,3591 & 2,82 & 0,0065 \\
D2008IV & $-4,2541$ & 0,3900 & $-10,9$ & 0,0000 \\
\hline $\mathrm{R}^{2}$ ajustado & 0,8930 & & & \\
\hline
\end{tabular}

Fuente: Elaboración propia.

\section{- $\quad$ Estimación MiDaS}

La estimación de los modelos MiDaS se realizó de manera recursiva, reestimando el modelo con cada nueva observación mensual disponible. Se estimó todas las especificaciones resultantes de combinar los siguientes criterios:

- Término autorregresivo: especificaciones con primer rezago de la variable dependiente (ADLMiDaS) y sin él.

- Rezagos de variable explicativa: especificaciones con 3, 6, 9 y 12 rezagos de la tasa de variación del IMAE.

- Polinomio de ponderación: especificaciones calculadas mediante seis formas funcionales para el polinomio de ponderación:

- $\quad$ Densidad beta normalizada con rezago final cero o diferente de cero.

- $\quad$ Polinomio de rezagos Almon exponencial normalizado.

- $\quad$ Coeficientes no restringidos (U-MIDAS).

- $\quad$ Polinomio con función de grada en los rezagos 3, 6, 9 y 12.

- $\quad$ Polinomio de rezagos de Almon de orden 3. 
Se decidió trabajar solamente con las estimaciones de cada caso obtenidas mediante el polinomio de Almon exponencial normalizado porque consistentemente mostraron mejor ajuste a los datos y pronósticos más acertados.

\section{- $\quad$ Estimación ARIMA}

En el ejercicio de comparación se incluyó pronósticos basados en modelos ARIMA con el fin de evaluar la ganancia de incluir información de más alta frecuencia con respecto a modelos que incluyen solamente información rezagada de la variable trimestral. Para ello se buscó la especificación ARIMA más adecuada a los datos de acuerdo con el algoritmo implementado en el paquete TRAMO/SEATS, cuyos detalles pueden consultarse en Gómez y Maravall (1996) y Gómez y Maravall (1994). La búsqueda de especificación ARIMA se repitió cada trimestre con los datos más actualizados disponibles ${ }^{12}$.

En 14 de las 16 estimaciones se seleccionó la especificación tipo "Airline Model" dada por $(0,1,1)(0,1,1)$, que corresponde a

$$
\Delta \Delta_{4} v p i b_{t}=(1+\theta B)\left(1+\Theta B^{4}\right) \varepsilon_{t}
$$

donde $\mathrm{B}$ es el operador de rezagos y $\varepsilon_{\mathrm{t}}$ es ruido blanco. Este modelo ha mostrado ser apropiado para modelar gran cantidad de series económicas. Ver Box y Jenkins (1970) para más detalles.

\section{- $\quad$ Esquema de pronóstico}

A partir de cada especificación estimada con los datos más actualizados, se calculó pronósticos $t+k$ con horizontes de $k=0,1,2,3$ y 4 trimestres, de manera que se obtuvieran series de 16 datos con un mismo horizonte cada una, para la muestra común 2009-III - 2013-II ${ }^{13}$. Todos los pronósticos con $k=0$ se calcularon para cada uno de los siguientes casos de disponibilidad de información mensual del IMAE:

- 0 meses conocidos

- 1 mes conocido

- 2 meses conocidos

- 3 meses conocidos

Para los restantes horizontes, los pronósticos bridge consideraron todos estos casos mientras que los MiDaS únicamente el de 3 meses conocidos.

\section{- Criterios para la evaluación de los pronósticos}

Mediante una prueba estándar se verificó que los pronósticos no fueran sesgados. La prueba consiste en estimar la regresión

$$
v p i b_{t}=\alpha_{0}+\alpha_{1} v \hat{p} i b_{t}+\varepsilon_{t}
$$

12 En ejercicios de evaluación con datos simulados, el algoritmo de TRAMO/SEATS ha mostrado buena capacidad para identificar correctamente la especificación de las series. Ver, entre otros, Maravall, López Pavón y Pérez Cañete (2012), y Hood, Ashley y Findley (2000).

13 Nótese que debido al esquema de publicación de cifras, de los pronósticos hechos en el trimestre $t$ para ese mismo trimestre $(k=0)$, solo los realizados con 0 y 1 meses conocidos corresponden estrictamente a pronósticos en tiempo real. 
y calcular el estadístico Wald para la hipótesis (conjunta) de que no hay sesgo, dada por $\left(\alpha_{0}=0, \alpha_{1}=1\right)$.

El ajuste de los pronósticos $v \hat{p} i b$ a los valores observados de la variación trimestral del PIB (vpib) se midió mediante la raíz del error cuadrático medio (RECM) y el error porcentual absoluto medio (EPAM):

$$
R E C M=\sqrt{\frac{1}{m} \sum_{i=1}^{m}\left(v p i b_{t+i}-v \hat{p} i b_{t+i}\right)^{2}} \quad \quad E P A M=\frac{1}{m} \sum_{i=1}^{m}\left|\frac{v p i b_{t+i}-v \hat{p} i b_{t+i}}{v p i b_{t+i}}\right|
$$

donde $m$ es el número de pronósticos.

Una práctica usual es calcular el cambio relativo en la RECM resultante de utilizar los modelos de frecuencia mixta, en comparación con modelos más sencillos, como un ARIMA. En este estudio, adicionalmente, se realizó pruebas de capacidad de pronóstico de Harvey, Leybourne y Newbold (1997), para identificar casos en los que el error cuadrático medio (ECM) es significativamente distinto entre pronósticos. El estadístico HLN es una modificación del estadístico DM de Diebold y Mariano (1995):

$$
H L N=\left(\frac{m+1-2 k+k(k-1) / m}{m}\right)^{1 / 2} D M
$$

donde la hipótesis nula es que los dos modelos siendo comparados tienen la misma capacidad de pronóstico (ECM no son significativamente distintos).

Como medida adicional de capacidad predictiva se calculó el porcentaje de acierto en la predicción del signo de vpib:

$$
A=\frac{\sum_{i=1}^{m} 1\left\{\operatorname{sgn}\left(\Delta v p i b_{t+i}\right)=\operatorname{sgn}\left(\Delta v \hat{p} i b_{t+i}\right)\right\}}{m}
$$

con la función 1\{.\} igual a 1 si el signo del cambio en el pronóstico es igual al del cambio en el valor efectivo de vpib.

\section{RESULTADOS DE LA EVALUACIÓN}

Los gráficos 1 a 5 en el anexo 3 muestran los pronósticos obtenidos, organizados por horizonte e información mensual disponible.

Los pronósticos MiDaS presentan un comportamiento muy similar entre horizontes y conjuntos de información. En general, siguen la evolución de la variación trimestral del PIB, con la excepción de la segunda mitad de 2012, cuando muestran una aceleración que no ocurrió, y parecen ajustar mejor que los otros pronósticos para los horizontes mayores a 0 . Resulta claro que los pronósticos bridge y ARIMA se ajustan menos al valor efectivo conforme aumenta el horizonte de pronóstico.

A partir de estos pronósticos, para cada caso se calculó una combinación de los tres pronósticos que mostraron mejor ajuste según su RECM. Para obtenerla se usó el método propuesto por Capistrán y Timmermann (2009), que consiste en calcular los valores ajustados de la regresión:

$$
y_{t+h}=\alpha+\beta \bar{y}_{t+h}+\varepsilon_{t+h}
$$


donde $\bar{y}_{t+h}$ es el promedio simple de los pronósticos siendo combinados y $y_{t+h}$ es el valor efectivo de la variable. Al incluir una constante, este método corrige posibles sesgos de los pronósticos individuales.

Los datos resultantes de aplicar los criterios de evaluación descritos en la sección 3 se presentan en los cuadros 2 a 11 organizados por horizonte de pronóstico y conjunto de información utilizado. La comparación del desempeño de los pronósticos combinados se realiza en el último apartado de esta sección.

\section{Verificación de sesgos}

El resultado más relevante es que para dos pronósticos, MiDaS con 9 y con 12 rezagos, no se rechaza la hipótesis nula de que no hay sesgo en todos los horizontes y conjuntos de información considerados. En general, los pronósticos MiDaS no autorregresivos son los que muestran mejor desempeño que los otros en este criterio a lo largo de todos los horizontes considerados, con 22 no rechazos de 28 posibles. Los MiDaS autorregresivos, con 4 excepciones, muestran evidencia de sesgo. De los pronósticos bridge, solo los calculados para horizontes de $k=0$ y $k=1$ son insesgados, $y$ en el caso de los ARMA solo el correspondiente a $\mathrm{k}=0$ lo es.

\section{Capacidad de pronóstico}

Es claro que el pronóstico ARMA es el que genera menores valores de la RECM y del EPAM para el horizonte $k=0$, pero esa ventaja disminuye conforme más información del trimestre se incluye en los pronósticos bridge y MiDaS. Por ejemplo, la RECM del ARMA es 37\% menor que la del bridge con 0 meses conocidos, pero si se compara con la del bridge con 3 meses esa diferencia disminuye a cerca de 24\%. Los pronósticos MiDaS simples a este horizonte superan a los de MiDas autorregresivos y tienden a converger a los pronósticos bridge cuando se considera toda la información mensual del trimestre.

A horizontes mayores, el ajuste de los modelos ARMA y bridge medido por la RECM y el EPAM se deteriora considerablemente. Esto es más notorio en los pronósticos ARMA, para los que esos indicadores se triplican en el mejor de los casos $(k=1)$. En contraste, los valores de la RECM y la EPAM correspondientes a pronósticos MiDAS se mantienen relativamente estables: la diferencia promedio entre los valores para $\mathrm{k}=4$ y $\mathrm{k}=0$ con 3 meses conocidos es de apenas $6,5 \%$. Además, esos indicadores resultan considerablemente menores a los del modelo ARMA ${ }^{14}$. De nuevo, los pronósticos de MiDaS simples muestran mejor desempeño que los de autorregresivos, aunque esta diferencia es menos evidente que en el caso de pronóstico para $k=0$.

Los resultados de las pruebas HLN, para la verificación de la significancia de las diferencias en los ECM, figuran en los cuadros 3, 5, 7, 9 y 11. Para pronósticos con un horizonte de k=0, el pronóstico ARMA es significativamente más preciso que todos los demás cuando se considera 0 o 1 meses del trimestre. Cuando los bridge y los MiDaS incluyen información de al menos 2 meses del trimestre, las diferencias en capacidad de pronóstico del ARMA son significativas solo con respecto a algunos de los pronósticos MiDaS autorregresivos. Por otra parte, a este horizonte las pruebas no muestran diferencias para modelos MiDaS con diferente número de rezagos, o entre modelos bridge y MiDaS simples, aunque éstos últimos sí evidencian una capacidad de pronóstico mayor a la de los MiDaS autorregresivos.

14 Por ejemplo, el promedio de la RECM para los MiDaS simples es entre 47\% y 72\% menor al del ARMA (41- 70\% para los autorregresivos). 
A horizontes mayores a 0 , todos los MiDaS son significativamente más precisos que los pronósticos ARMA y los bridge. Además, los MiDaS simples con diferente longitud de rezago de nuevo generan pronósticos que no difieren entre sí, pero que sí son significativamente más precisos que los de MiDaS autorregresivos. En particular, los MiDaS de 9 y 12 rezagos de nuevo superan consistentemente a los MiDaS autorregresivos.

Con respecto a la capacidad para predecir la dirección de los cambios en la tasa de variación del producto, al horizonte $\mathrm{k}=0$ los pronósticos ARMA son los que se desempeñan mejor, con un porcentaje de acierto de $86,67 \%$, pero esa capacidad se deteriora considerablemente a horizontes mayores (porcentaje de acierto entre 20\% y 40\% para horizontes de 2, 3 y 4 trimestres). Este mismo patrón se aprecia para los pronósticos bridge, que presentan un porcentaje de acierto por encima de 73\% para pronóstico en tiempo real, que disminuye a horizontes mayores.

Por el contrario, el porcentaje de acierto de los pronósticos MiDaS se mantiene consistentemente alto: por encima del $66 \%$ para horizontes de 0 y 1 trimestres y por encima de $73 \%$ para horizontes de 2, 3 y 4 trimestres. Los MiDaS simples y los autorregresivos tienen un desempeño similar, con promedios de acierto entre versiones con diferente número de rezagos de alrededor del $78 \%$ para horizontes diferentes a 0 . Las versiones no autorregresivas muestran promedios de acierto marginalmente más altos en los horizontes $\mathrm{k}=3$ y $\mathrm{k}=4$.

\section{Combinación de pronósticos}

En el cuadro 12 se detalla la composición de las combinaciones de pronósticos calculadas, y en los cuadros 13 y 14 figuran los resultados de la evaluación. El pronóstico ARIMA forma parte de todas las combinaciones calculadas para pronóstico con horizonte 0 , mientras que el bridge entra en dos de esas combinaciones. Todas las combinaciones para horizontes mayores a 0 están conformadas por MiDaS simples de 6, 9 y 12 rezagos. Debe destacarse que para el caso de pronóstico en tiempo real ( $k=0,1$ mes conocido), la combinación se compone incluye los tres tipo de modelo analizados.

Por construcción, las combinaciones mediante el método de Capistrán y Timmerman generan pronósticos insesgados. Además, al usarse los tres pronósticos con mejor ajuste en cada caso, es esperable que las combinaciones resultaran en RECM y EPAM menores a los de todos los pronósticos no incluidos en ellas. La combinación que muestra el mejor ajuste es la de pronósticos con toda la información mensual del trimestre para el horizonte 0, con una RECM y un EPAM de 0,15 p.p y $13 \%$, respectivamente. En el resto de casos, esos indicadores promedian 0,19 p.p y 15\%.

Las pruebas HLN muestran que para el horizonte de 0, las combinaciones son significativamente más precisas que todos los pronósticos que la integran, excepto para el bridge con 1 mes conocido. Para horizontes mayores a 0 , la precisión de las combinaciones no difiere significativamente de la de ninguno de sus componentes, $y$ en general solo supera a la de los bridge y a la de algunos pronósticos MiDaS autorregresivos en los horizontes más bajos. Debe destacarse que las combinaciones resultan significativamente más precisas que los pronósticos ARIMA a todos los horizontes considerados.

El acierto en la predicción de la dirección de cambios en la tasa de variación del producto es $80 \%$, excepto uno con 93\%. Esto supera la mayoría de pronósticos, salvo algunos incluidos en la combinación, como el MiDaS de 6 rezagos, que consistentemente muestra un acierto de 87\% para horizontes mayores a 0 . 
CUADRO 2

EVALUACIÓN: INSESGAMIENTO E INDICADORES DE CAPACIDAD DE PRONÓSTICO HORIZONTE K=0

\begin{tabular}{|c|c|c|c|c|}
\hline & RECM & EPAM & $\begin{array}{l}\text { Acierto cambios } \\
\text { en dirección }\end{array}$ & $\begin{array}{l}\text { Insesgamiento } \\
\mathrm{P}(\text { Wald }) 1\end{array}$ \\
\hline \multicolumn{5}{|l|}{0 meses conocidos } \\
\hline Bridge & 0,2779 & 0,2529 & 0,7333 & 0,0377 \\
\hline ARIMA & 0,1751 & 0,1387 & 0,8667 & 0,2460 \\
\hline \multicolumn{5}{|l|}{1 mes conocido } \\
\hline Bridge & 0,2448 & 0,2298 & 0,8000 & 0,0729 \\
\hline MiDaS, 3 rezagos & 0,2859 & 0,2470 & 0,7333 & 0,0860 \\
\hline MiDaS, 6 rezagos & 0,2677 & 0,2177 & 0,6667 & 0,3555 \\
\hline MiDaS, 9 rezagos & 0,2859 & 0,2454 & 0,7333 & 0,1122 \\
\hline MiDaS, 12 rezagos & 0,2850 & 0,2479 & 0,7333 & 0,1230 \\
\hline MiDaS, autorregresivo, 3 rezagos & 0,3148 & 0,2717 & 0,6667 & 0,0275 \\
\hline MiDaS, autorregresivo, 6 rezagos & 0,2955 & 0,2388 & 0,7333 & 0,1245 \\
\hline MiDaS, autorregresivo, 9 rezagos & 0,3212 & 0,2781 & 0,7333 & 0,0329 \\
\hline MiDaS, autorregresivo, 12 rezagos & 0,3232 & 0,2829 & 0,7333 & 0,0314 \\
\hline \multicolumn{5}{|l|}{2 meses conocidos } \\
\hline Bridge & 0,2368 & 0,2250 & 0,7333 & 0,0745 \\
\hline MiDaS, 3 rezagos & 0,2565 & 0,2306 & 0,7333 & 0,0703 \\
\hline MiDaS, 6 rezagos & 0,2104 & 0,1778 & 0,8000 & 0,2887 \\
\hline MiDaS, 9 rezagos & 0,2335 & 0,2128 & 0,7333 & 0,2130 \\
\hline MiDaS, 12 rezagos & 0,2401 & 0,2198 & 0,7333 & 0,1752 \\
\hline MiDaS, autorregresivo, 3 rezagos & 0,2889 & 0,2630 & 0,7333 & 0,0200 \\
\hline MiDaS, autorregresivo, 6 rezagos & 0,2489 & 0,2231 & 0,8667 & 0,0587 \\
\hline MiDaS, autorregresivo, 9 rezagos & 0,2709 & 0,2558 & 0,8000 & 0,0608 \\
\hline MiDaS, autorregresivo, 12 rezagos & 0,2817 & 0,2674 & 0,8000 & 0,0385 \\
\hline \multicolumn{5}{|l|}{3 meses conocidos } \\
\hline Bridge & 0,2295 & 0,2195 & 0,7333 & 0,0653 \\
\hline MiDaS, 3 rezagos & 0,2530 & 0,2156 & 0,7333 & 0,0644 \\
\hline MiDaS, 6 rezagos & 0,2342 & 0,2046 & 0,8667 & 0,0396 \\
\hline MiDaS, 9 rezagos & 0,2221 & 0,1935 & 0,7333 & 0,1110 \\
\hline MiDaS, 12 rezagos & 0,2309 & 0,2068 & 0,8000 & 0,0723 \\
\hline MiDaS, autorregresivo, 3 rezagos & 0,2645 & 0,2267 & 0,8000 & 0,0441 \\
\hline MiDaS, autorregresivo, 6 rezagos & 0,2835 & 0,2497 & 0,8667 & 0,0032 \\
\hline MiDaS, autorregresivo, 9 rezagos & 0,2559 & 0,2240 & 0,7333 & 0,0251 \\
\hline MiDaS, autorregresivo, 12 rezagos & 0,2665 & 0,2469 & 0,7333 & 0.016 \\
\hline
\end{tabular}

1 En negrita no rechazo de hipótesis de insesgamiento, al 5\% de significancia.

Fuente: elaboración propia. 


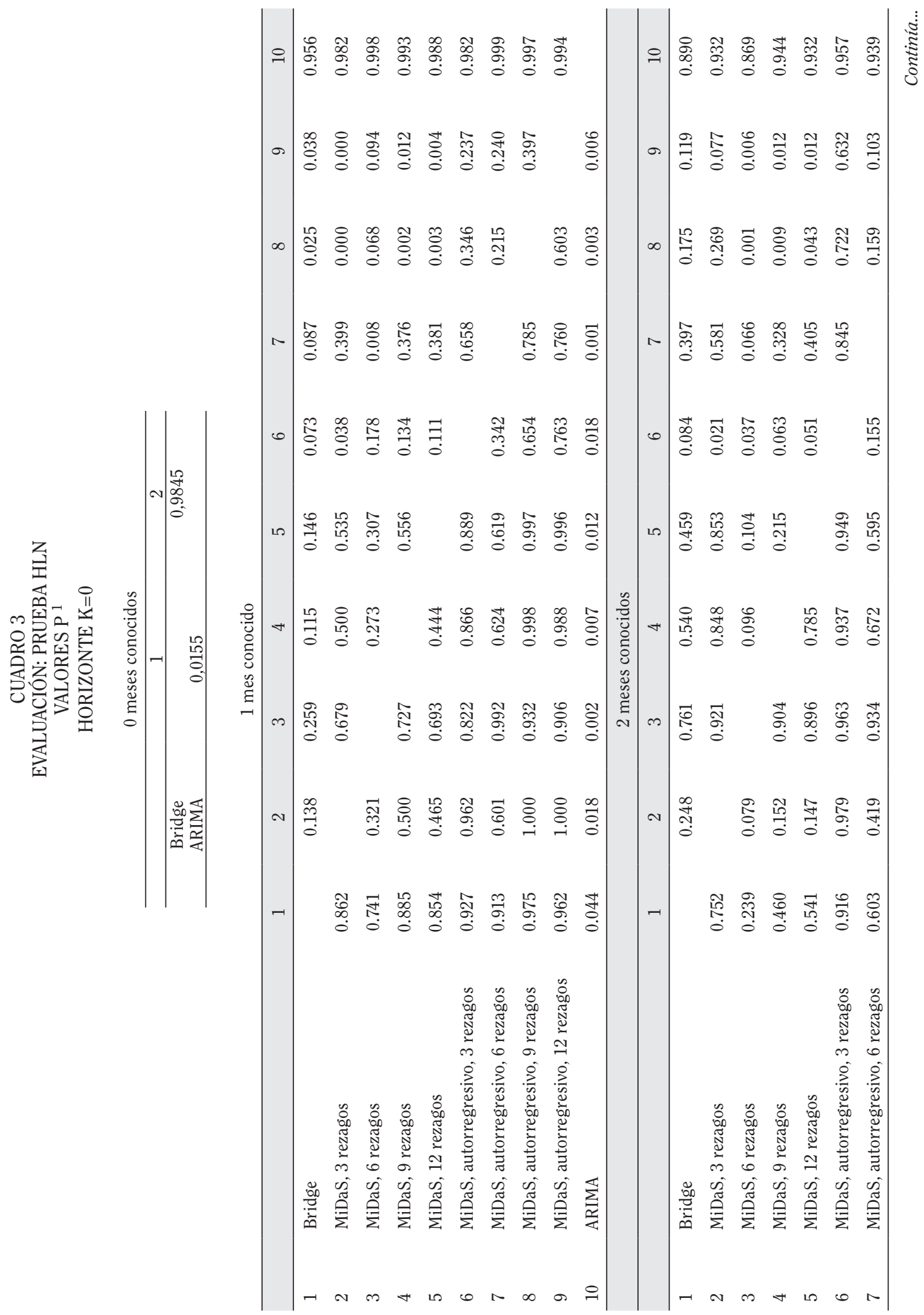




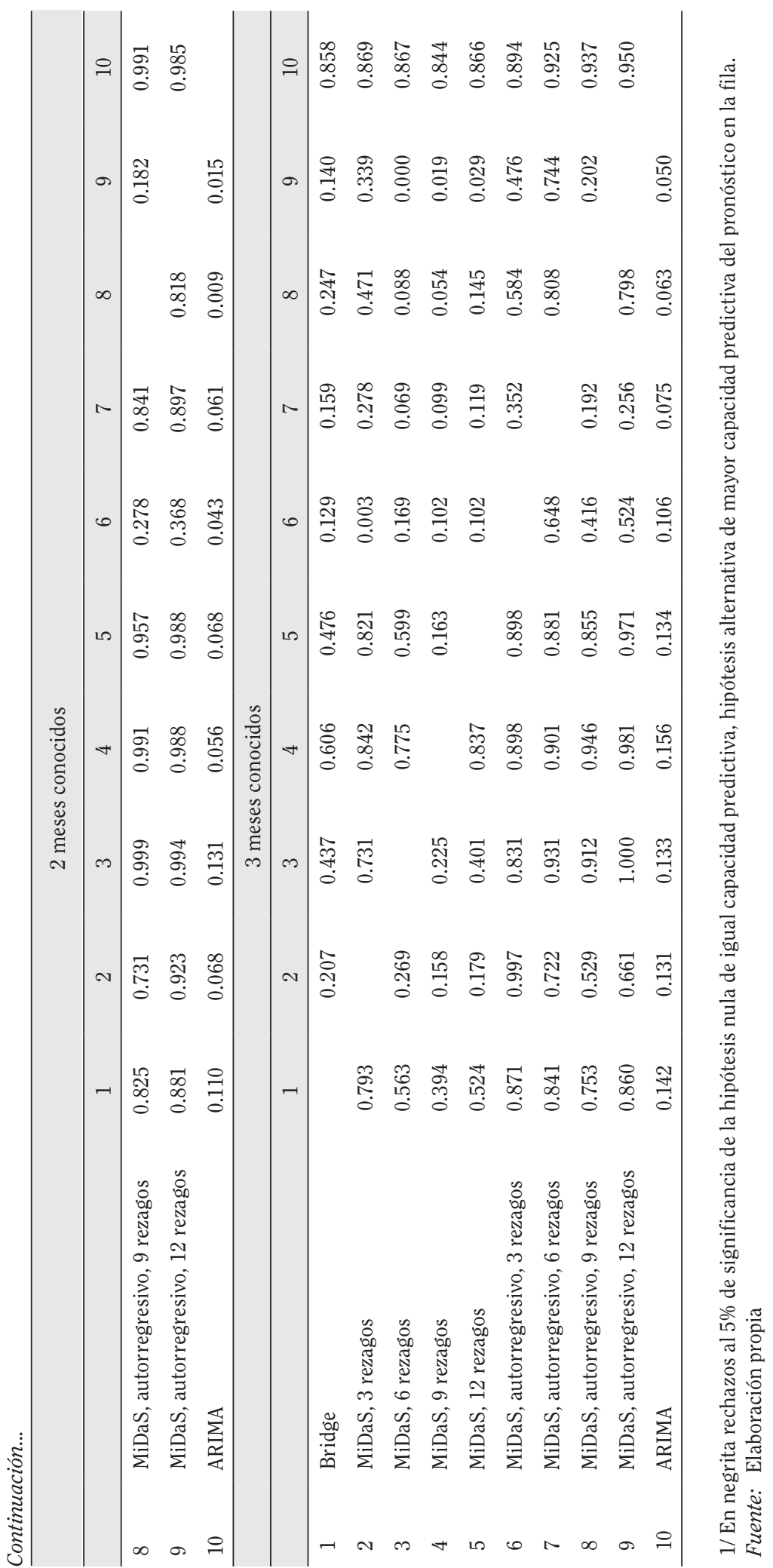




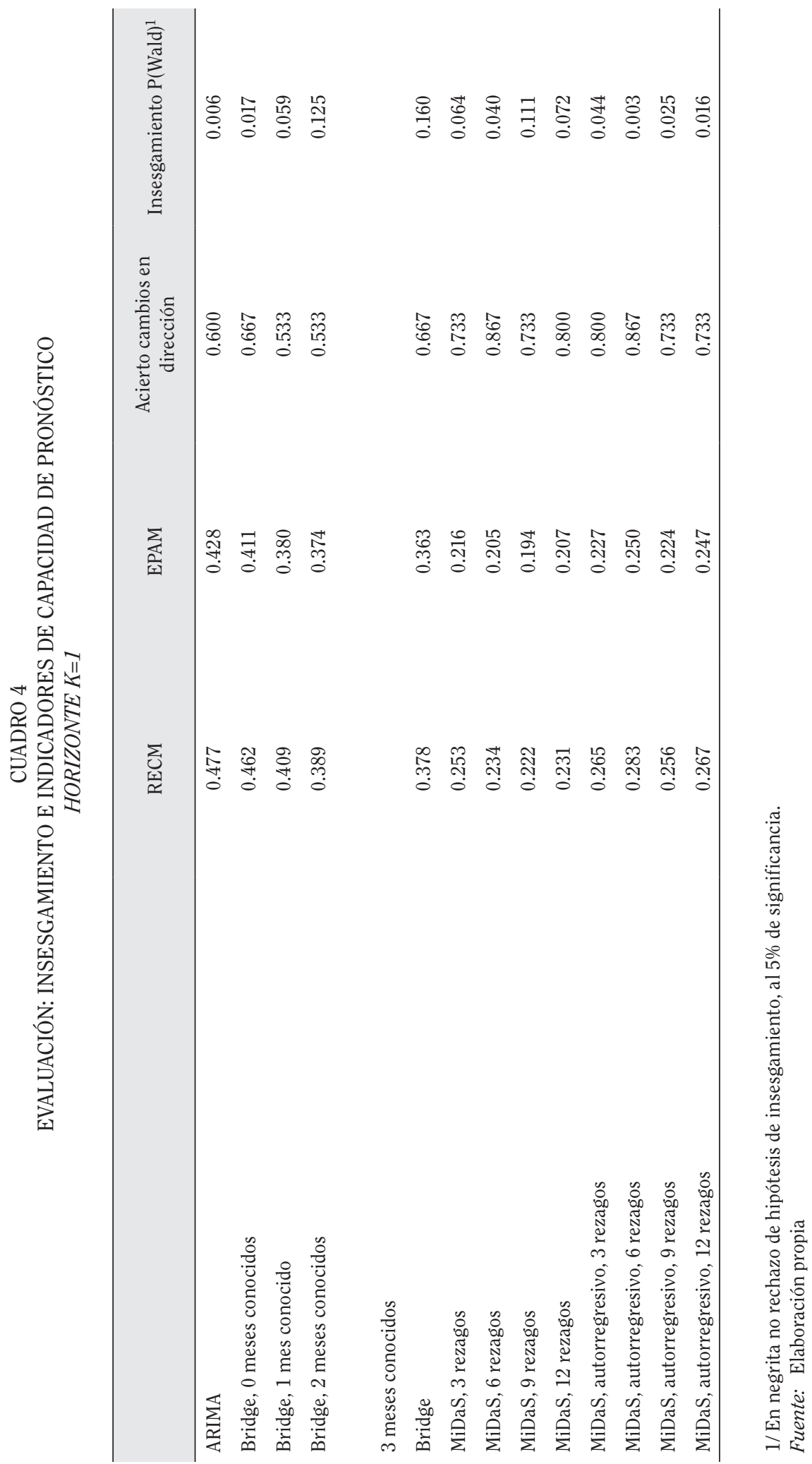




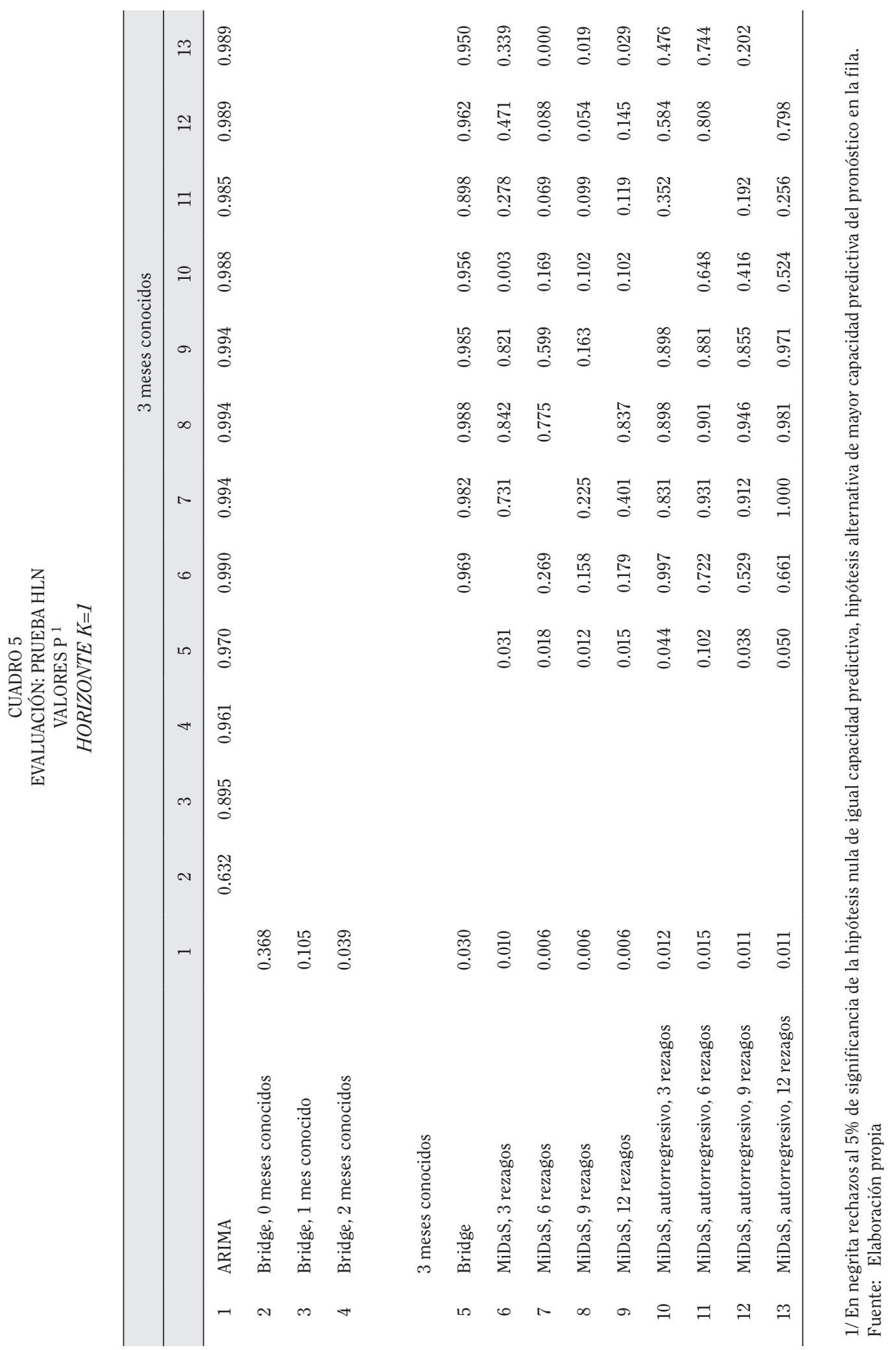


CUADRO 6

EVALUACIÓN: INSESGAMIENTO E INDICADORES DE CAPACIDAD DE PRONÓSTICO HORIZONTE K=2

\begin{tabular}{|c|c|c|c|c|}
\hline & RECM & EPAM & $\begin{array}{c}\text { Acierto cambios en } \\
\text { dirección }\end{array}$ & $\begin{array}{c}\text { Insesgamiento } \\
\mathrm{P}(\text { Wald })^{1}\end{array}$ \\
\hline ARIMA & 0.684 & 0.676 & 0.400 & 0.000 \\
\hline Bridge, 0 meses conocidos & 0.918 & 0.756 & 0.400 & 0.000 \\
\hline Bridge, 1 mes conocido & 0.845 & 0.636 & 0.400 & 0.000 \\
\hline Bridge, 2 meses conocidos & 0.732 & 0.603 & 0.533 & 0.000 \\
\hline \multicolumn{5}{|c|}{3 meses conocidos } \\
\hline Bridge & 0.591 & 0.550 & 0.400 & 0.000 \\
\hline MiDaS, 3 rezagos & 0.254 & 0.217 & 0.733 & 0.059 \\
\hline MiDaS, 6 rezagos & 0.235 & 0.206 & 0.867 & 0.038 \\
\hline MiDaS, 9 rezagos & 0.224 & 0.196 & 0.733 & 0.098 \\
\hline MiDaS, 12 rezagos & 0.234 & 0.209 & 0.800 & 0.064 \\
\hline
\end{tabular}

1/ En negrita no rechazo de hipótesis de insesgamiento, al 5\% de significancia.

Fuente: Elaboración propia 


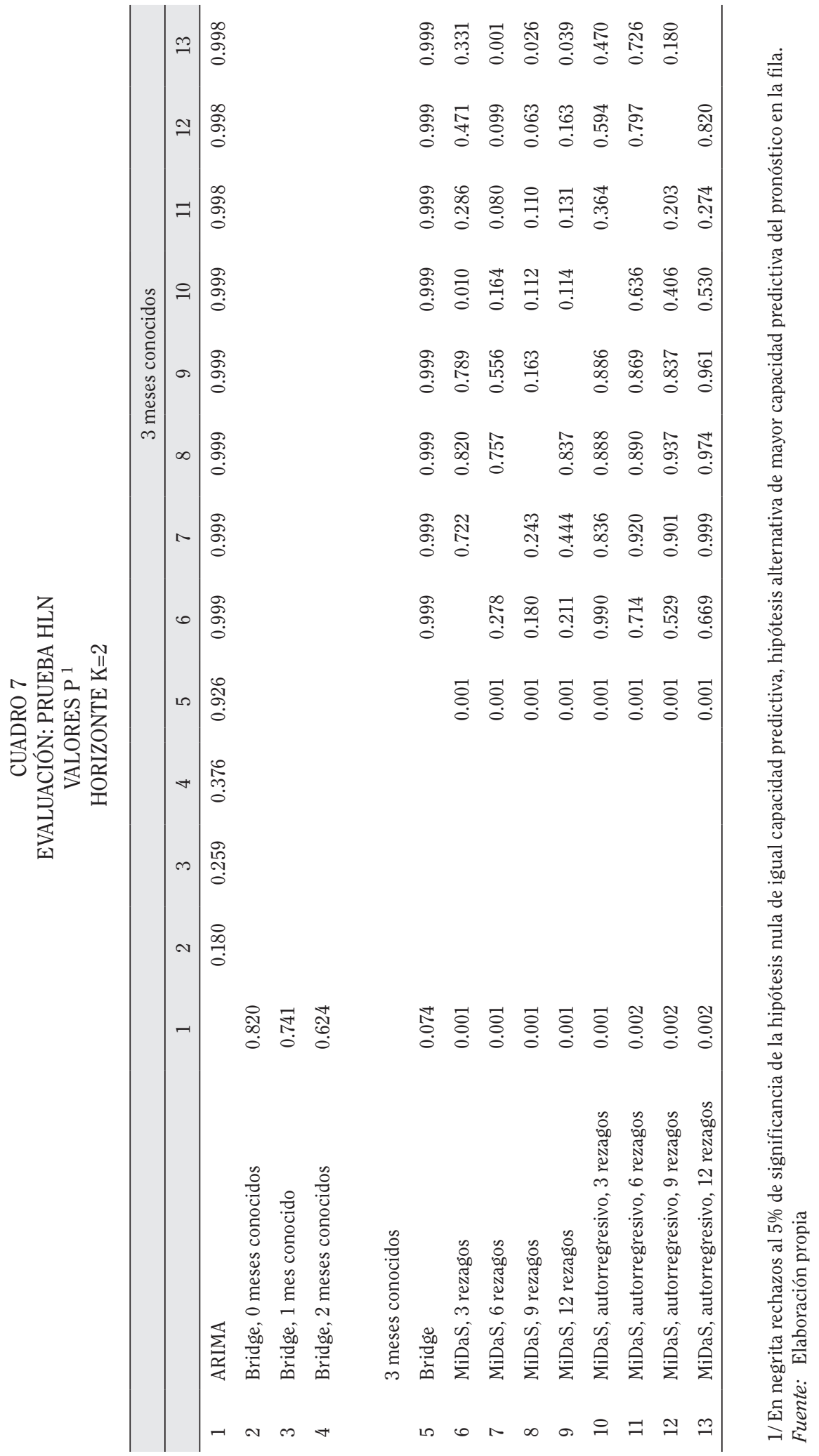




\section{CUADRO 8 \\ EVALUACIÓN: INSESGAMIENTO E INDICADORES DE CAPACIDAD DE PRONÓSTICO HORIZONTE K=3}

\begin{tabular}{|c|c|c|c|c|}
\hline & RECM & EPAM & $\begin{array}{c}\text { Acierto cambios } \\
\text { en dirección }\end{array}$ & $\begin{array}{c}\text { Insesgamiento } \\
\mathrm{P}(\text { Wald }) 1\end{array}$ \\
\hline ARIMA & 0.784 & 0.776 & 0.200 & 0.000 \\
\hline Bridge, 0 meses conocidos & 1.094 & 0.863 & 0.467 & 0.000 \\
\hline Bridge, 1 mes conocido & 1.051 & 0.807 & 0.467 & 0.000 \\
\hline Bridge, 2 meses conocidos & 1.011 & 0.810 & 0.400 & 0.000 \\
\hline \multicolumn{5}{|l|}{3 meses conocidos } \\
\hline Bridge & 0.833 & 0.718 & 0.267 & 0.000 \\
\hline MiDaS, 3 rezagos & 0.257 & 0.218 & 0.733 & 0.049 \\
\hline MiDaS, 6 rezagos & 0.241 & 0.208 & 0.867 & 0.029 \\
\hline MiDaS, 9 rezagos & 0.226 & 0.197 & 0.800 & 0.088 \\
\hline MiDaS, 12 rezagos & 0.237 & 0.210 & 0.800 & 0.055 \\
\hline MiDaS, autorregresivo, 3 rezagos & 0.264 & 0.228 & 0.733 & 0.042 \\
\hline MiDaS, autorregresivo, 6 rezagos & 0.280 & 0.250 & 0.933 & 0.004 \\
\hline MiDaS, autorregresivo, 9 rezagos & 0.257 & 0.225 & 0.733 & 0.025 \\
\hline MiDaS, autorregresivo, 12 rezagos & 0.268 & 0.248 & 0.733 & 0.015 \\
\hline
\end{tabular}

1/ En negrita no rechazo de hipótesis de insesgamiento, al 5\% de significancia.

Fuente: Elaboración propia 


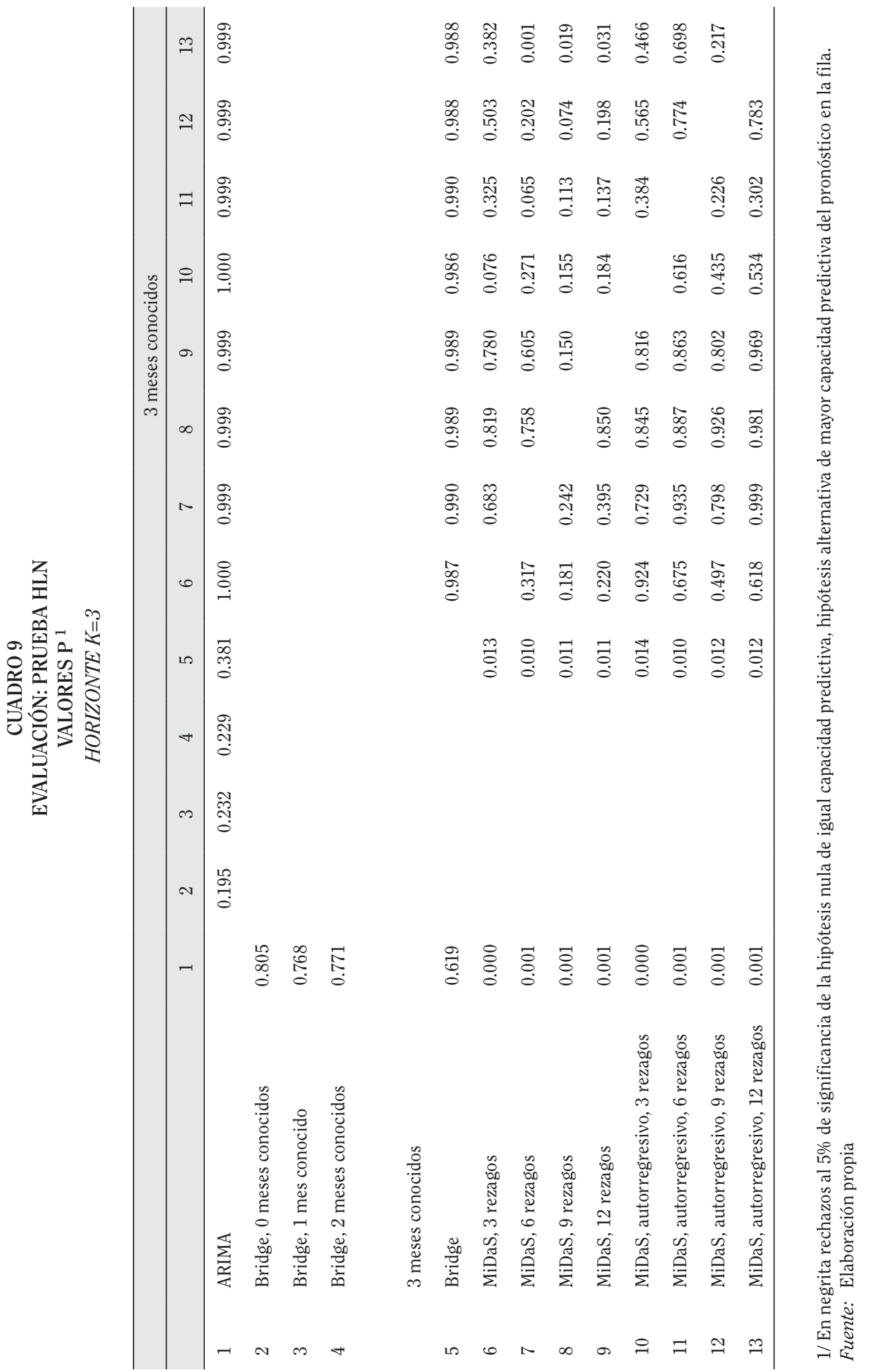




\section{CUADRO 10 \\ EVALUACIÓN: INSESGAMIENTO E INDICADORES DE CAPACIDAD DE PRONÓSTICO HORIZONTE K=4}

\begin{tabular}{|c|c|c|c|c|}
\hline & RECM & EPAM & $\begin{array}{l}\text { Acierto cambios } \\
\text { en dirección }\end{array}$ & $\begin{array}{l}\text { Insesgamiento } \\
\mathrm{P}(\text { Wald }) 1\end{array}$ \\
\hline ARIMA & 0.832 & 0.790 & 0.333 & 0.000 \\
\hline Bridge, 0 meses conocidos & 0.941 & 0.839 & 0.267 & 0.000 \\
\hline Bridge, 1 mes conocido & 0.909 & 0.810 & 0.333 & 0.000 \\
\hline Bridge, 2 meses conocidos & 0.925 & 0.826 & 0.200 & 0.000 \\
\hline \multicolumn{5}{|l|}{3 meses conocidos } \\
\hline Bridge & 0.818 & 0.737 & 0.200 & 0.000 \\
\hline MiDaS, 3 rezagos & 0.253 & 0.216 & 0.733 & 0.060 \\
\hline MiDaS, 6 rezagos & 0.238 & 0.207 & 0.867 & 0.035 \\
\hline MiDaS, 9 rezagos & 0.229 & 0.198 & 0.800 & 0.081 \\
\hline MiDaS, 12 rezagos & 0.234 & 0.209 & 0.800 & 0.064 \\
\hline MiDaS, autorregresivo, 3 rezagos & 0.256 & 0.222 & 0.733 & 0.069 \\
\hline MiDaS, autorregresivo, 6 rezagos & 0.260 & 0.243 & 0.933 & 0.011 \\
\hline MiDaS, autorregresivo, 9 rezagos & 0.254 & 0.224 & 0.733 & 0.031 \\
\hline MiDaS, autorregresivo, 12 rezagos & 0.257 & 0.243 & 0.733 & 0.028 \\
\hline
\end{tabular}

1/ En negrita no rechazo de hipótesis de insesgamiento, al 5\% de significancia.

Fuente: Elaboración propia 


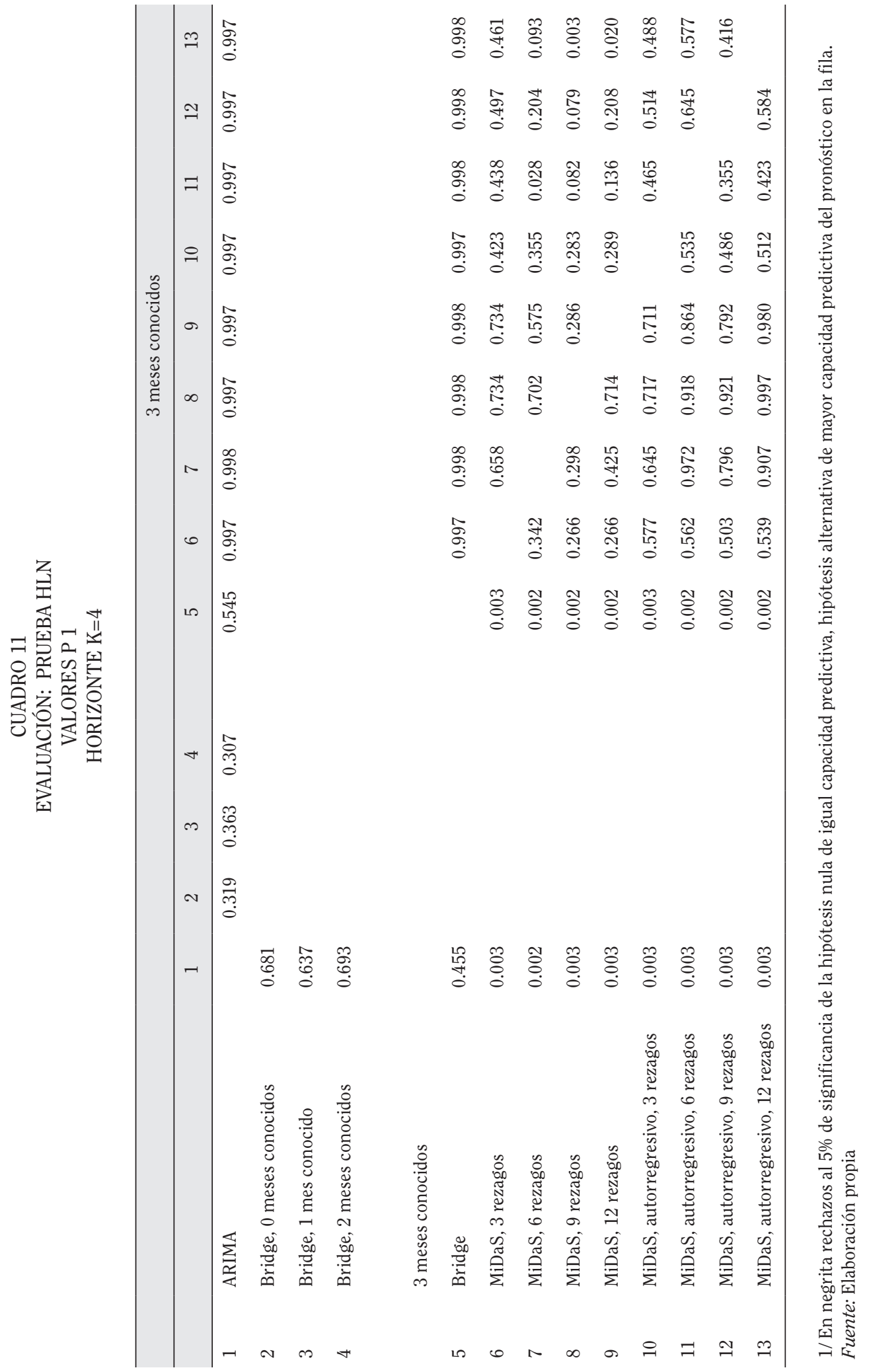



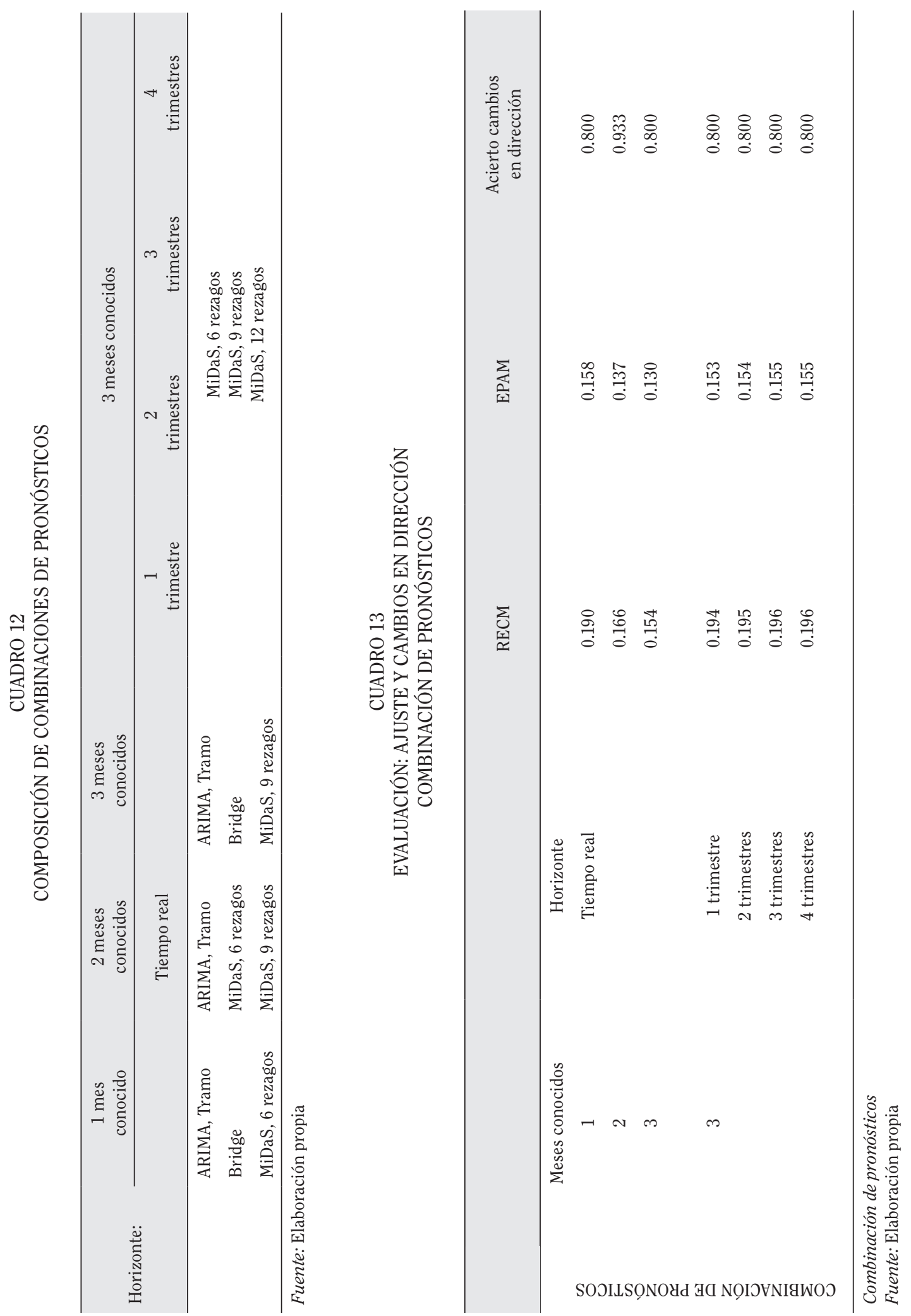


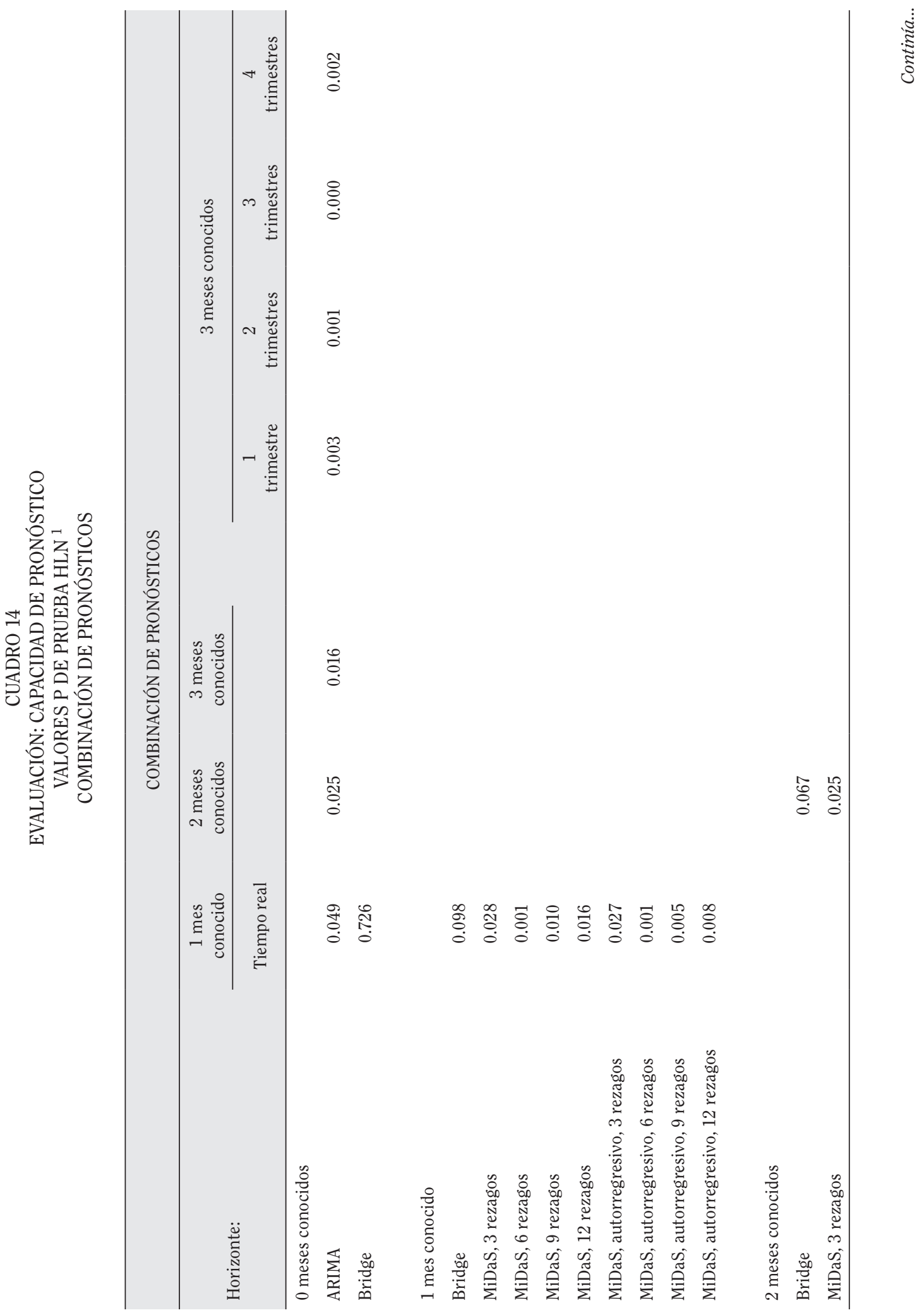




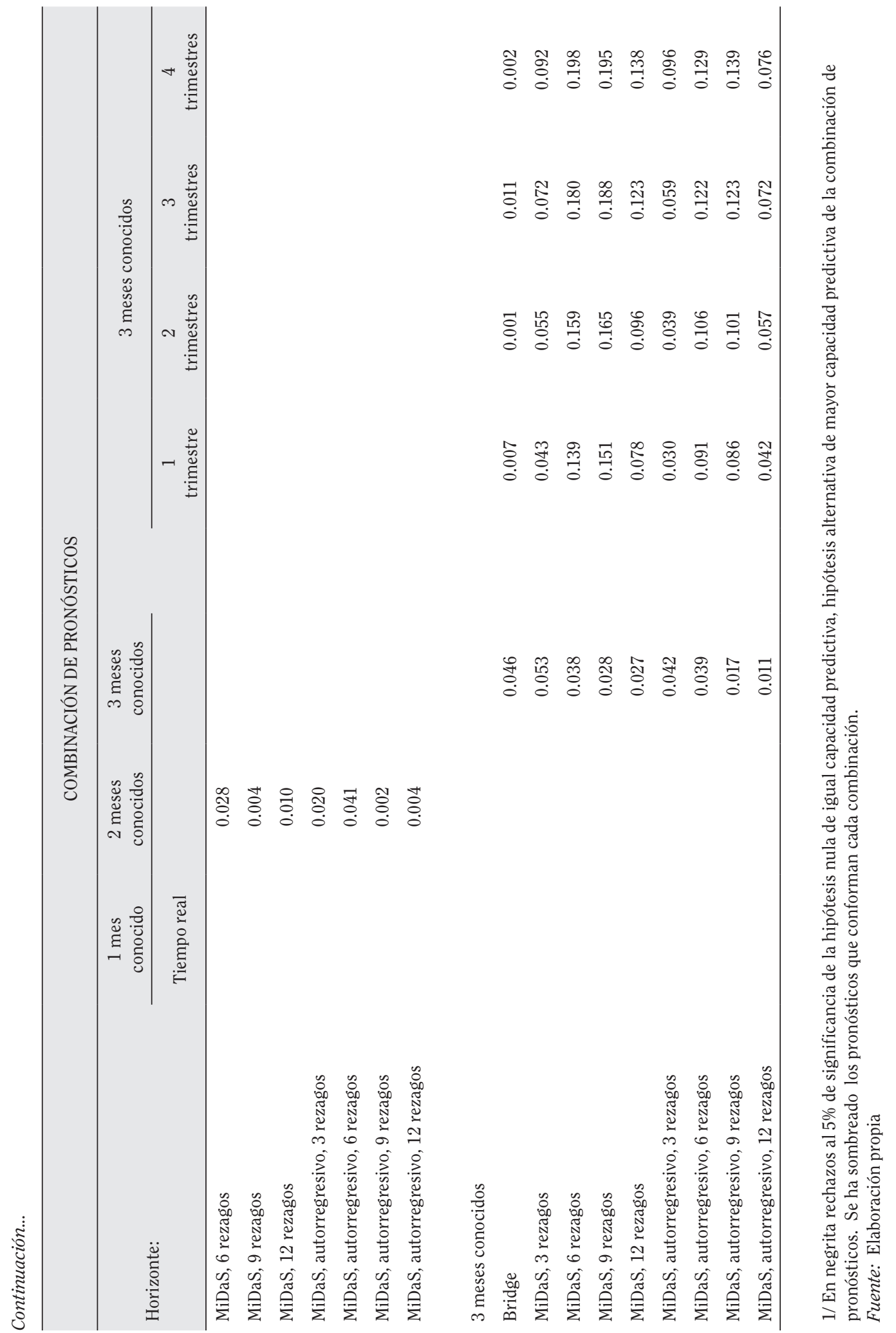




\section{CONCLUSIONES}

Un banco central requiere contar oportunamente con estimaciones del crecimiento del producto, con las cuales pueda anticipar la formación de presiones inflacionarias. Por esta razón, los modelos de frecuencia mixta han ganado popularidad como herramientas de pronóstico, ya que permiten incorporar nueva información disponible más rápidamente.

Se considera que existe una ganancia en oportunidad y en precisión al utilizar los modelos de frecuencia mixta para obtener proyecciones del crecimiento con antelación a la publicación de cuentas nacionales. El ejercicio de evaluación realizado en este estudio mostró que la inclusión de información de variables de más alta frecuencia que la variable pronosticada mejora la precisión con respecto a pronósticos univariados, especialmente a horizontes más largos. Además, en la mayoría de casos considerados, la calidad de los pronósticos mejora conforme más información mensual del trimestre vigente se utilice para la proyección.

En general, los pronósticos MiDaS mostraron el mejor desempeño: al incrementarse el horizonte de pronóstico, sus RECM y EPAM aumentaron relativamente poco, su porcentaje de acierto de cambios en la tasa de variación del producto se mantuvo estable y varios de ellos continuaron siendo insesgados. En particular, los pronósticos obtenidos mediante MiDaS simples con 9 y 12 rezagos resultaron insesgados para todos los horizontes y conjuntos de información evaluados, $y$ son los que mostraron más diferencias significativas con los ARIMA, los bridge y los demás MiDaS.

Si bien llama la atención que, a diferencia de lo usual en la literatura, los pronósticos bridge no mostraran mejor desempeño que los ARIMA, debe recordarse las limitaciones existentes para contar con indicadores mensuales que estén disponibles durante el trimestre correspondiente. Esto resulta en modelos bridge basados en un solo indicador, cuando lo usual es que se utilice un conjunto más amplio de información. Sin embargo, para pronóstico en tiempo real (casos k=0 con 0 y 1 meses del trimestre), la capacidad de pronóstico de los modelos bridge es similar a la de los MiDaS simples pero significativamente mayor a la de algunos MiDaS autorregresivos.

Combinar los pronósticos con mejor ajuste resulta útil sobre todo para proyectar al horizonte $k=0$, que incluye pronóstico en tiempo real, pues en esos casos se aprecia una diferencia significativa en la capacidad de pronóstico en comparación con los integrantes de la combinación.

Los resultados de este trabajo muestran la conveniencia de utilizar modelos de frecuencia mixta para pronosticar la variación del producto real en Costa Rica. En concreto, se recomienda dar continuidad al pronóstico de dicha variable mediante las combinaciones de pronósticos descritas en el cuadro 12 para el caso de pronóstico en tiempo real, y mediante los pronósticos MiDaS simples con 9 y 12 rezagos para los restantes horizontes. Así mismo, se considera aconsejable evaluar pronósticos obtenidos mediante otras técnicas de este tipo, como los VAR de frecuencia mixta (MFVAR) y los modelos de factores de frecuencia mixta.

\section{REFERENCIAS}

Antipa, P., Barhoumi, K., Brunhes-Lesange, V., \& Darné, O. (2012). Nowcasting German GDP: A Comparison of Bridge and Factor Models (Document de Travail No. 401). Banque de France.

Armesto, M. T., Engemann, K. M., \& Owyang, M. T. (2010). Forecasting with Mixed Frequencies. Federal Reserve Bank of St. Louis Review, 92(6), 521-36.

Barhoumi, K., Brunhes-Lesange, V., Darné, O., Ferrara, L., Pluyaud, B., \& Rouvreau, B. (2008). Monthly Forecasting of French GDP: A Revised Version of the OPTIM Model (Notes d'Études et de Recherche 222). Banque de France.

Barhoumi, K., Darné, O., Ferrara, L., \& Pluyaud, B. (2012). Monthly GDP Forecasting Using Bridge

Models: Application for the French Economy. Bulletin of Economic Research, 64, s57-s70.

Baumeister, C., Guérin, P., \& Kilian, L. (2014). Do High-Frequency Financial Data Help Forecast Oil 
Prices? The MIDAS Touch at Work (Working Paper / Document de travail 2014-11). Bank of Canada.

BCCR. (s.f). Metodología de cálculo del Índice Mensual de Actividad Económica (IMAE). Disponible en: http://indicadoreseconomicos.bccr.fi.cr/indicadoreseconomicos/Documentos// DocumentosMetodologiasNotasTecnicas/Metodología\%20de\%20cálculo\%20del\%20IMAE.htm

Box, G. E., \& Pierce, D. A. (1970). Distribution of Residual Autocorrelations in Autoregressive Integrated Moving Average Time Series Models. Journal of the American Statistical Association, 65 (332), 1509-1526.

Box, G., \& Jenkins, G. (1970). Time Series Analysis: Forecasting and Control. San Francisco: Holden-Day.

Bulligan, G., Marcellino, M., \& Venditti, F. (2012). Forecasting Economic Activity with Higher Frequency Targeted Predictors (Temi di Discussione 847). Banca d'Italia.

Capistrán, C., \& Timmermann, A. (2009). Forecast Combination With Entry and Exit of Experts. Journal of Business \& Economic Statistics, 27(4), 428-440.

Chen, X., \& Ghysels, E. (2011). News good or bad and its impact on volatility predictions over multiple horizons. Review of Financial Studies, 24(1), 46-81.

Chow, G. C. (1960). Tests of Equality Between Sets of Coefficients in Two Linear Regressions. Econometrica, 28(3), 591-605.

Clements, M. P., \& Galvão, A. B. (2006). Macroeconomic Forecasting with Mixed Frequency Data: Forecasting US output growth and inflation. (U. o. Department of Economics, Ed.) (Warwick Economic Research Papers No 773).

Cobb, M., Echavarría, G., Filippi, P., García, M., Godoy, C., González, W., y otros. (2011). Short-term GDP Forecasting Using Bridge Models: A Case for Chile (Documentos de Trabajo No. 626). Banco Central de Chile.

Diebold, F. X., \& Mariano, R. (1995). Comparing predictive accuracy. Journal of Business and Economic Statistics, 13, 253-265.

Doornik, J., \& Hansen, H. (1994). A Practical test for Univariate and Multivariate Normality (Discussion Paper). Nuffield College, Oxford University.

Doornik, J., \& Hendry, D. F. (1996). Empirical Econometric Modelling Using PcGive 9 for Windows. Londres: Timberlake Consultants Press.

Doornik, J., \& Hendry, D. F. (2007). PcGive 12. Londres: Timberlake Consultants Press.

Engle, R., Ghysels, E., \& Sohn, B. (2013). Stock market volatility and macroeconomic fundamentals. Review of Economics and Statistics, 95(3), 776-797.

Ghysels, E. (2012). Macroeconomics and the reality of mixed frequency data. SSRN: http://ssrn. com/abstract=2069998orhttp://dx.doi.org/10.2139/ssrn.2069998.

Ghysels, E. (2014). Matlab Toolbox for Mixed Sampling Frequency Data Analysis usin MIDAS Regression Models. Disponible en http://www.unc.edu/ eghysels/papers/MIDAS_Usersguide_ V1.0.pdf.

Ghysels, E., Santa-Clara, P., \& Valkanov, R. (2004). The MIDAS Touch: Mixed Data Sampling Regression Models. CIRANO Working Papers 2004s-20.

Ghysels, E., Sinko, A., \& Valkanov, R. (2006). MIDAS Regressions: Further Results and New Directions. Disponible en SSRN: http://ssrn.com/abstract=885683 or http://dx.doi. org/10.2139/ssrn.885683.

Godfrey, L. G. (1978). Testing for Higher-order Serial Correlation in Regression Equations When the Regressors Include Lagged Dependent Variables. Econometrica, 46(6), 1303-1313.

Golinelli, R., \& Parigi, G. (2007). The Use of Monthly Indicators to Forecast Quarterly GDP in the Short Run: An Application to the G7 Countries. Journal of Forecasting, 26(2), 77-94.

Gómez, V., \& Maravall, A. (1994). Prediction and Interpolation for Nonstationary Series with the Kalman Filter. Journal of the American Statistical Association, 89, 611-624. 
Gómez, V., \& Maravall, A. (1996). Programs TRAMO and SEATS, Instruction for User (Beta Version: september 1996). Banco de España Working Papers 9628.

Harvey, D., Leybourne, S., \& Newbold, P. (1997). Testing the equality of prediction mean squared errors. International Journal of Forecasting, 13, 281-291.

Hendry, D. F. (1979). Predictive Failure and Econometric Modelling in Macroeconomics: The Transactions Demand for Money. En P. Ormerod (Ed.), Economic Modelling (págs. 217-242). Londres: Heinemann.

Hood, C. C., Ashley, J. D., \& Findley, D. F. (2000). An Empirical Evaluation fo the Performance of TRAMO/SEATS on Simulated Series. Proceedings of the American Statistical Association, Business and Economics Section, 171-176.

Hoover, K. D., \& Perez, S. J. (1999). Data Mining Reconsidered: Encompassing and the General-tospecific Approach to Specification Search. Econometric Journal, 2(2), 167-191.

Ingenito, R., \& Trehan, B. (1996). Using Monthly Data to Predict Quarterly Output. Economic Review(3), 3-11.

Jarque, C. M., \& Bera, A. K. (1980). Efficient Tests for Normality, Homoscedasticity and Serial Independence of Regression Residuals. Economic Letters, 6(3), 255-259.

Klein, L. R., \& Sojo, E. (1989). Combinations of High and Low Frequency Data in Macroeconometric Models. En L. R. Klein, \& J. Márquez, Economics in Theory and Practice: An Eclectic Approach (págs. 3-16). Dordrecht: Kluwer.

Krolzig, H.-M., \& Hendry, D. F. (2001). Computer Automation of General-to-specific Model Selection Procedures. Journal of Economic Dynamics and Control, 25(6-7), 831-866.

Krolzig, H.-M., \& Hendry, D. F. (2005). The Properties of Automatic GETS Modelling. The Economic Journal, 115(502), C32-C61.

Kuzin, V., Marcellino, M., \& Schumacher, C. (2011). MIDAS vs. mixed-frequency VAR: Nowcasting GDP in the euro area. International Journal of Forecasting, 27, 529-542.

Maravall, A., López-Pavón, R., \& Pérez-Cañete, D. (2012). Reliability of the Automatic Identification of ARIMA Models in Program TRAMO. Presentado en el CEMFI Econometrics Workshop, 5 junio de 2012. Madrid, España.

Nicholls, D. F., \& Pagan, A. R. (1983). Heteroskedasticity in Models with Lagged Dependent Variables. Econometrica, 51(4), 1233-1242.

Parigi, G., \& Shlitzer, G. (1995). Quarterly Forecasts of the Italian Business Cycle by Means of Monthly Economic Indicators. Journal of Forecasting, 14, 117-141.

Trehan, B. (1992). Predicting Contemporaneous Output. Federal Reserve Bank of San Francisco Economic Review(2), 3-11.

White, H. (1980). A Heteroskedastic-consistent Covariance Matrix Estimator and A Direct Test for Heteroskedasticity. Econometrica, 48(4), 817-838.

Winkelried, D. (2012). Predicting quarterly aggregates with monthly indicators. (Serie Documentos de Trabajo DT. N 2012-023). Banco Central de Reserva del Perú. 


\title{
ANEXOS
}

\section{ANEXO 1 \\ COMPOSICIÓN DEL ÍNDICE MENSUAL DE ACTIVIDAD ECONÓMICA}

\author{
CUADRO 15 \\ ÍNDICES POR INDUSTRIA DEL IMAE
}

\author{
1. Agricultura, silvicultura y pesca \\ 2. Industria manufacturera \\ 3. Extracción de minas y canteras \\ 4. Electricidad $y$ agua \\ 5. Construcción \\ 6. Comercio \\ 7. Hoteles \\ 8. Transporte, almacenamiento y comunicaciones \\ 9. Servicios financieros y seguros \\ 10. Otros servicios prestados a empresas \\ 11. Servicios intermediación financiera medidos indirectamente \\ 12. Resto de industrias: Restaurantes, Actividades Inmobiliarias, Servicios de Administración Pública y \\ Servicios Comunales, Sociales y Personales
}

Fuente: BCCR. 


\section{Prueba de cambio estructural de Bai y Perron (2003)}

La prueba se aplicó a la serie de variaciones trimestrales del PIB real (tendencia-ciclo), con datos para el periodo 1991-II a 2013-II, $y$ a la serie de variaciones mensuales del IMAE (tendenciaciclo), con datos para ene-1991 a sep-2013. Se utilizó el código para Matlab escrito por Yohei Yamamoto, disponible en el sitio en Internet de Pierre Perron: http://people.bu.edu/perron/code.html. Se consideró un máximo de 5 quiebres, con un porcentaje de truncamiento de $15 \%$ para el cálculo de los estadísticos y de los valores críticos. Los cuadros 16, 17 y 18 muestran los resultados. Tanto con los estadísticos supF como con los UDmax no es posible rechazar en ningún caso la hipótesis nula de ausencia de quiebres estructurales en las series. El criterio de información LWZ selecciona un modelo sin quiebres como el que presenta mejor ajuste a los datos para ambas series, como también el criterio BIC para el caso de la tasa de variación del PIB.

\section{CUADRO 16
ESTADÍSTICO SUPF, PRUEBA DE CAMBIO ESTRUCTURAL DE BAI-PERRON}

\begin{tabular}{|c|c|c|c|c|c|}
\hline \multirow{2}{*}{$\begin{array}{c}\text { Hipótesis nula } \\
\text { vs } \\
\text { hipótesis alternativa }\end{array}$} & \multicolumn{2}{|c|}{ Estadístico de prueba } & \multicolumn{3}{|c|}{ Valores críticos } \\
\hline & Tasa variac. PIB real & Tasa variac. IMAE & $\mathrm{Al} 1 \%$ & $\mathrm{Al} \mathrm{5 \%}$ & Al 10\% \\
\hline 0 quiebres vs 1 quiebre & 2,48 & 0,49 & 12,29 & 8,58 & 7,04 \\
\hline 0 quiebres vs 2 quiebres & 5,29 & 2,45 & 9,36 & 7,22 & 6,28 \\
\hline 0 quiebres vs 3 quiebres & 4,31 & 1,41 & 7,60 & 5,96 & 5,21 \\
\hline 0 quiebres vs 4 quiebres & 2,66 & 1,74 & 6,19 & 4,99 & 4,41 \\
\hline 0 quiebres vs 5 quiebres & 2,51 & 0,91 & 4,91 & 3,91 & 3,47 \\
\hline
\end{tabular}

Fuente: Elaboración propia.

CUADRO 17

ESTADÍSTICO UDMAX, PRUEBA DE CAMBIO ESTRUCTURAL DE BAI-PERRON

\begin{tabular}{lcccc}
\hline & & \multicolumn{3}{c}{ Valores críticos } \\
\cline { 3 - 4 } Serie & Estadístico de prueba ${ }^{1 /}$ & $\mathrm{Al} \mathrm{1 \%}$ & $\mathrm{Al} \mathrm{5 \%}$ & $\mathrm{Al} \mathrm{10 \%}$ \\
\cline { 3 - 5 } Tasa variac. PIB real & 5,29 & & & 7,46 \\
Tasa variac. IMAE & 2,45 & 12,37 & 8,88 & \\
\hline
\end{tabular}

1/ Hipótesis nula: ausencia de quiebres. Alternativa: número desconocido de quiebres.

Fuente: Elaboración propia. 
CUADRO 18

CRITERIOS DE INFORMACIÓN, PRUEBA DE CAMBIO ESTRUCTURAL DE BAI-PERRON

\begin{tabular}{|c|c|c|c|c|}
\hline & \multicolumn{2}{|c|}{ Tasa variac. PIB real } & \multicolumn{2}{|c|}{ Tasa variac. IMAE } \\
\hline & $\mathrm{BIC}$ & LWZ & BIC & LWZ \\
\hline 0 quiebres & $-0,02641^{*}$ & $-0,01511^{*}$ & 0.2942 & $0.2979 *$ \\
\hline 1 quiebre & 0,00492 & 0,09565 & 0.2883 & 0.3405 \\
\hline 2 quiebres & 0,05629 & 0,22699 & 0.2897 & 0.3904 \\
\hline 3 quiebres & 0,06449 & 0,31573 & $0.2507^{*}$ & 0.3999 \\
\hline 4 quiebres & 0,13354 & 0,46591 & 0.2666 & 0.4645 \\
\hline 5 quiebres & 0,26753 & 0,68166 & 0.3508 & 0.5973 \\
\hline
\end{tabular}

* Indica el número de quiebres elegido por el BIC (criterio de información bayesiano, o de Schwarz) o por el LWZ (criterio de información de Liu, Wu y Zidek).

Fuente: Elaboración propia.

\section{Pruebas de raíz unitaria}

Si bien el gráfico de las series sugiere utilizar un modelo con constante y sin tendencia en las pruebas de raíz unitaria, se decidió realizar todas las variantes de las pruebas y comparar el ajuste de la ecuación de prueba. En las pruebas Dickey-Fuller aumentadas se utilizó selección automática de la longitud de rezago con base en el criterio de información de Schwarz considerando hasta 11 rezagos para tasa de variación PIB real y hasta 15 rezagos para tasa de variación del IMAE. En las pruebas Phillips-Perron se utilizó kernel de Bartlett para estimar el espectro, con selección automática del ancho de banda (bandwidth) mediante Newey-West. Los cuadros 19 y 20 muestran los resultados. En todos los casos puede rechazarse la hipótesis nula de raíz unitaria al 1\% de significancia.

\section{CUADRO 19
ESTADÍSTICOS DE LA PRUEBA DICKEY-FULLER AUMENTADA (ADF)}

\begin{tabular}{lcc}
\hline Modelo & Tasa variac. PIB real & Tasa variac. IMAE \\
\hline Constante & $-5,80^{* \dagger}$ & $-3,75^{* \dagger}$ \\
& $(0$ rezagos $)$ & $(11$ rezagos $)$ \\
Constante y tendencia & $-5,87^{*}$ & $-3,74^{* *}$ \\
Sin ambos & $(0$ rezagos $)$ & $(11$ rezagos $)$ \\
\end{tabular}

* Rechazo de la hipótesis nula de raíz unitaria al 1\% de significancia (** rechazo al 5\%).

$\dagger$ Indica el modelo con el mejor ajuste.

Fuente: Elaboración propia. 
CUADRO 20

ESTADÍSTICOS DE LA PRUEBA PHILLIPS-PERRON (PP)

\begin{tabular}{lcc}
\hline Modelo & Tasa variac. PIB real & Tasa variac. IMAE \\
\hline Constante & $-5,86^{* \dagger}$ & $-4,99^{* \dagger}$ \\
\multirow{2}{*}{ Constante $y$ tendencia } & (Bandwidth: 3) & (Bandwidth: 11) \\
\multirow{2}{*}{ Sin ambos } & $-5,94^{*}$ & $-5,03^{*}$ \\
& $($ Bandwidth: 3$)$ & (Bandwidth: 11) \\
& $-3,09^{*}$ & $-3,02^{*}$ \\
\hline
\end{tabular}

* Rechazo de la hipótesis nula de raíz unitaria al 1\% de significancia (** rechazo al 5\%).

$\dagger$ Indica el modelo con el mejor ajuste.

Fuente: Elaboración propia. 


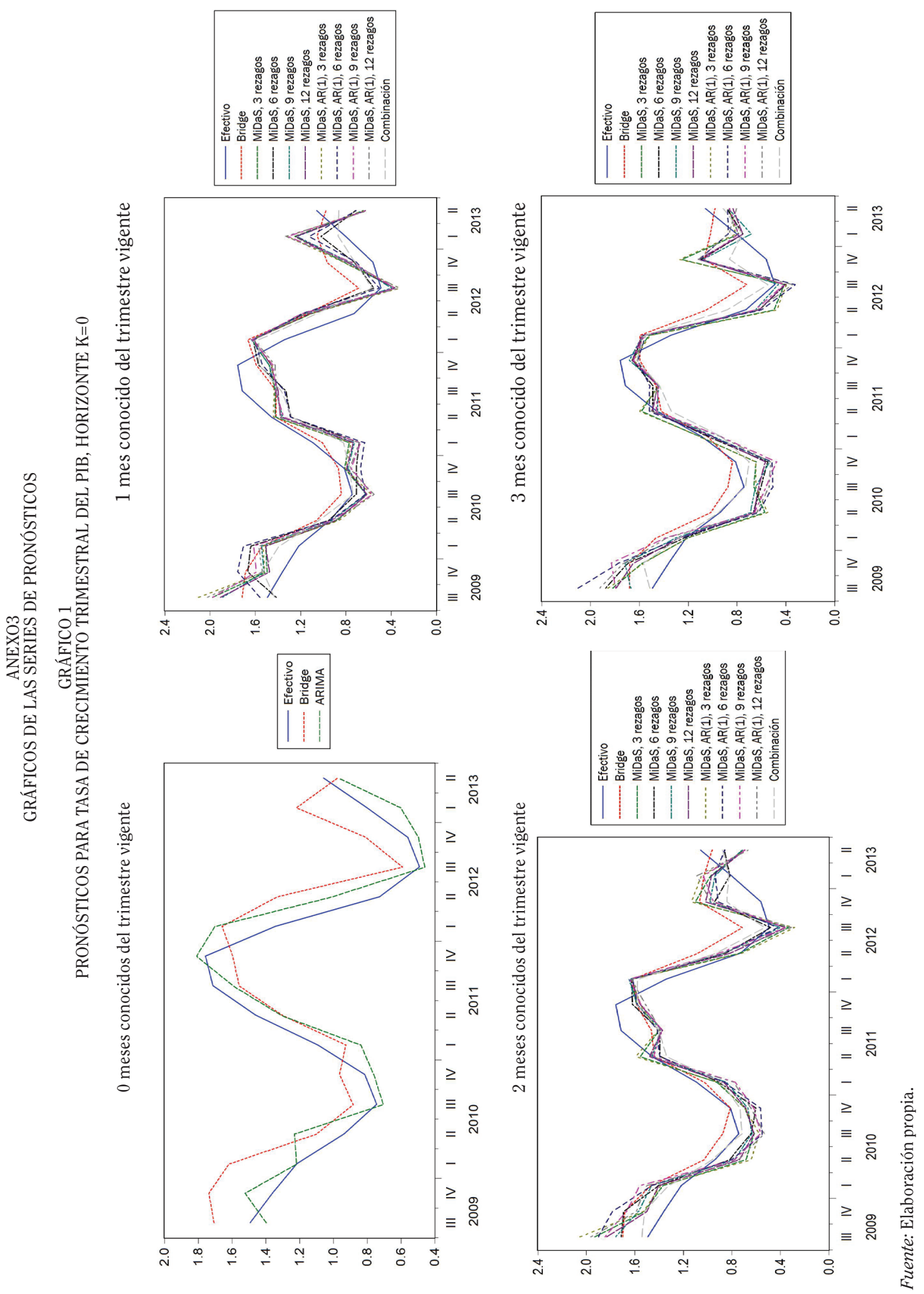




\section{GRÁFICO 2 \\ PRONÓSTICOS PARA TASA DE CRECIMIENTO TRIMESTRAL DEL PIB, HORIZONTE K=1}

\section{$0,1,2$ meses conocidos del trimestre vigente}

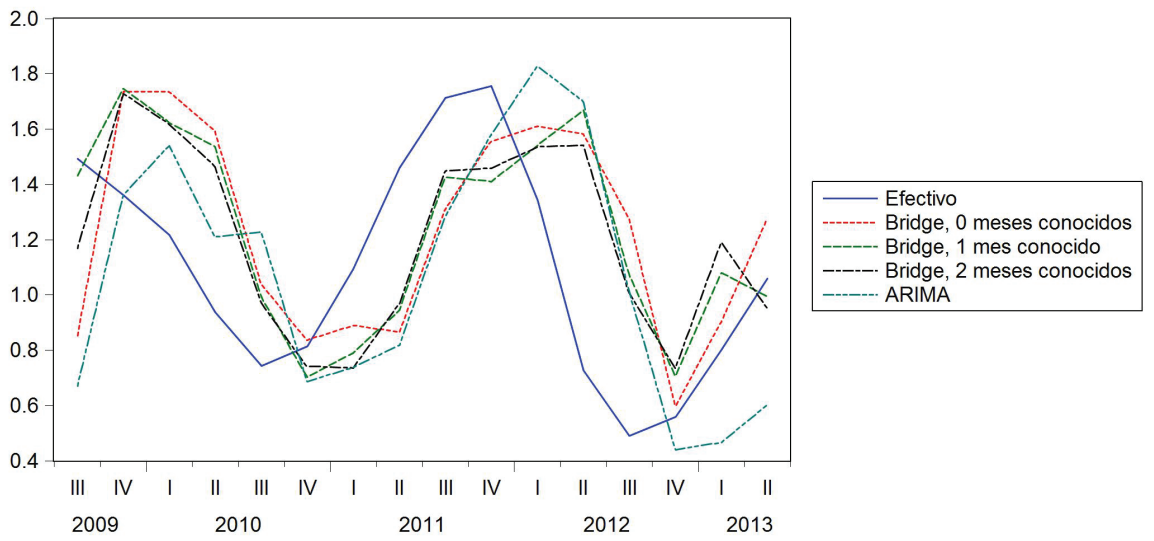

3 mes conocido del trimestre vigente

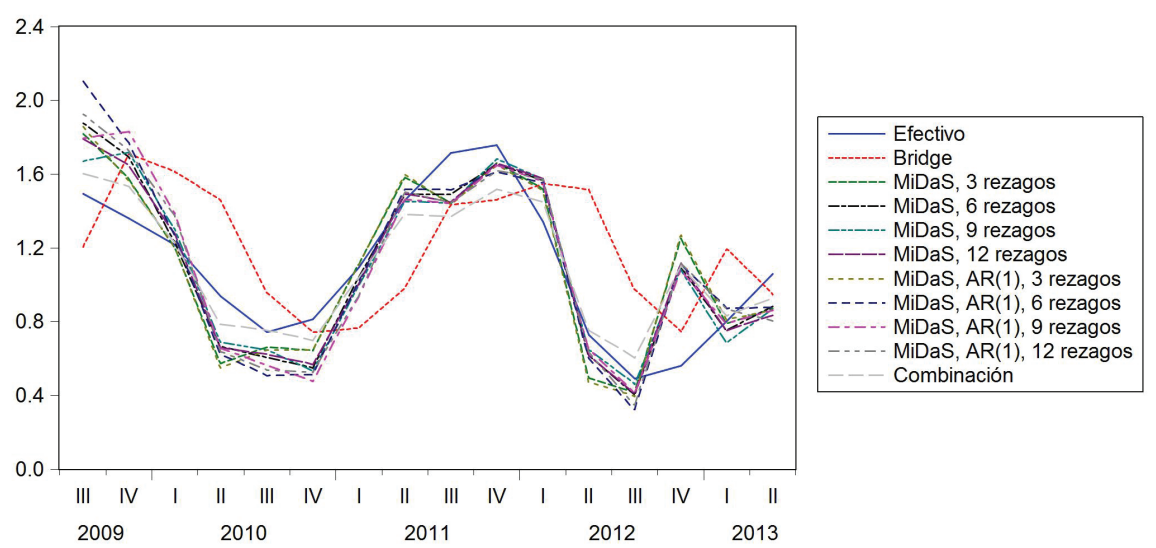

Fuente: Elaboración propia. 


\section{GRÁFICO 3}

PRONÓSTICOS PARA TASA DE CRECIMIENTO TRIMESTRAL DEL PIB, HORIZONTE K=2

\section{$0,1,2$ meses conocidos del trimestre vigente}

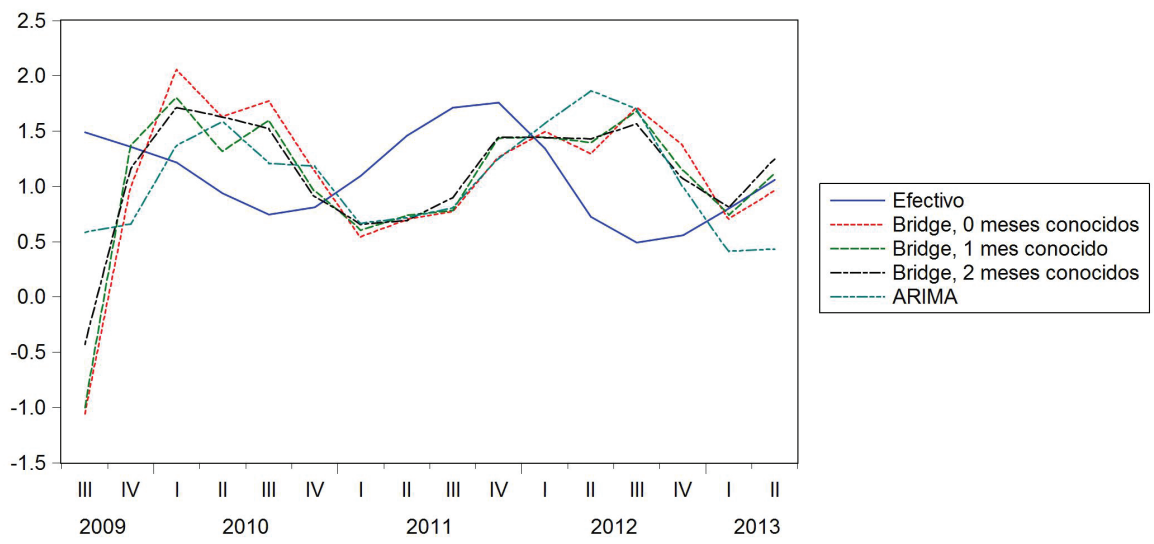

3 meses conocidos del trimestre vigente

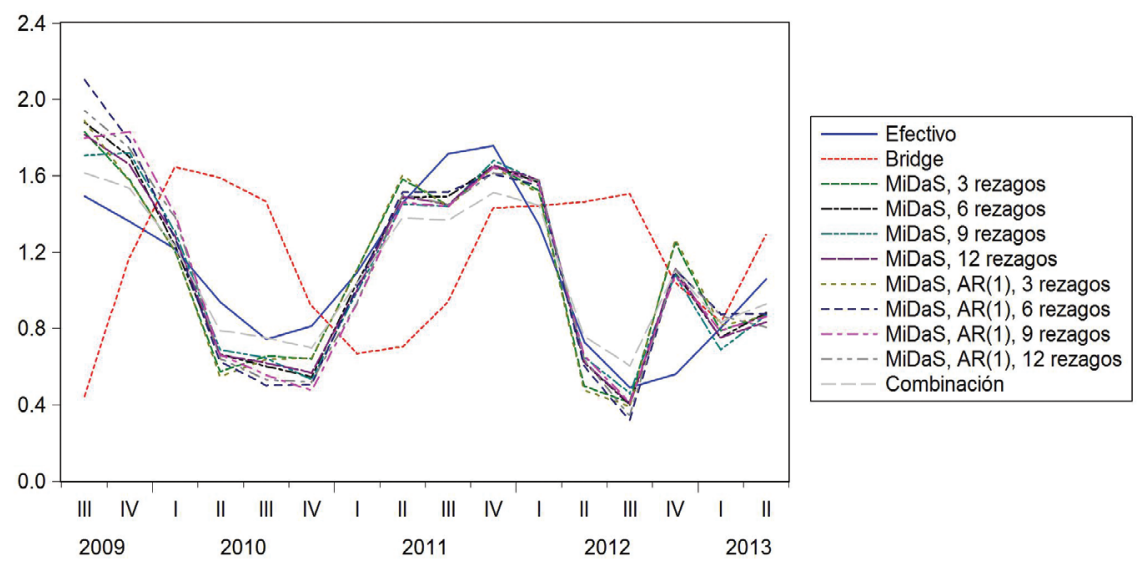

Fuente: Elaboración propia. 


\section{GRÁFICO 4}

PRONÓSTICOS PARA TASA DE CRECIMIENTO TRIMESTRAL DEL PIB, HORIZONTE K=3

0,1,2 meses conocidos del trimestre vigente

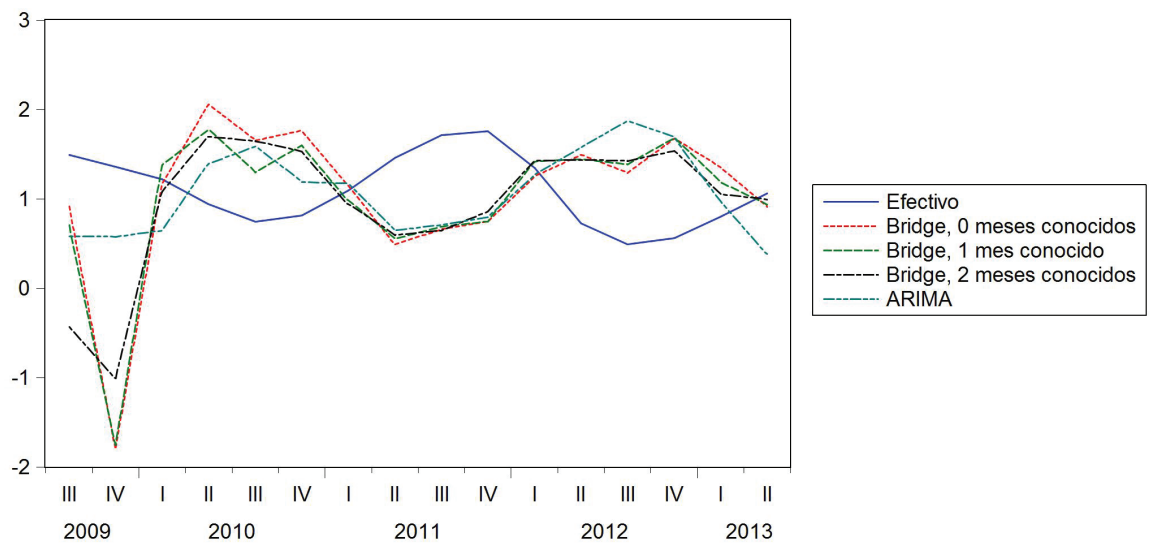

3 meses conocidos del trimestre vigente

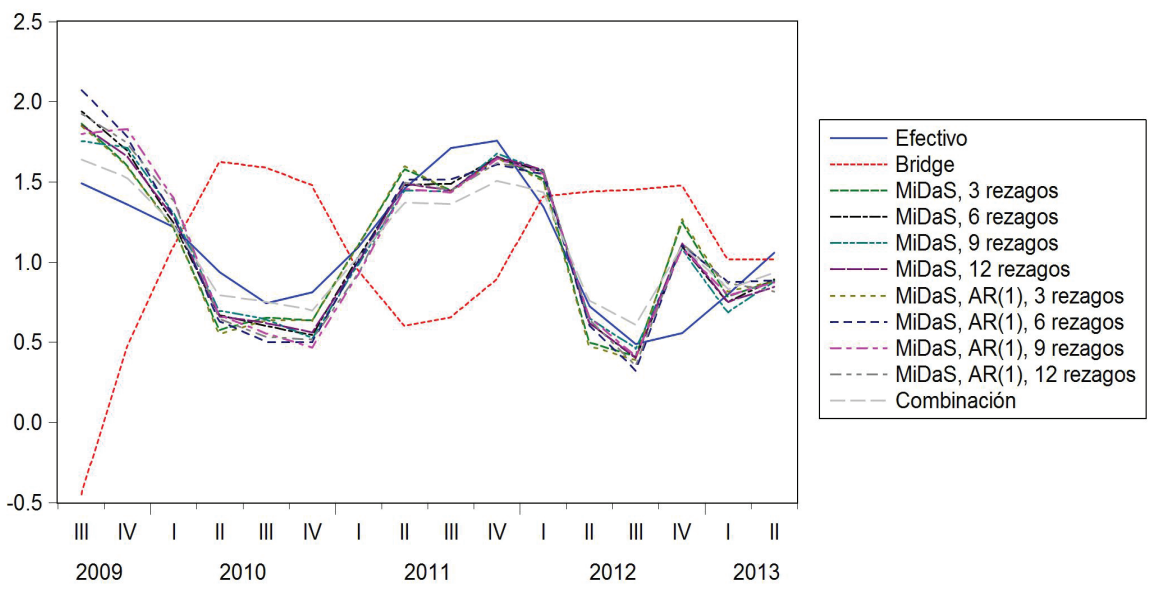

Fuente: Elaboración propia. 


\section{GRÁFICO 5 \\ PRONÓSTICOS PARA TASA DE CRECIMIENTO TRIMESTRAL DEL PIB, HORIZONTE K=4}

\section{0, 1, 2 meses conocidos del trimestre vigente}
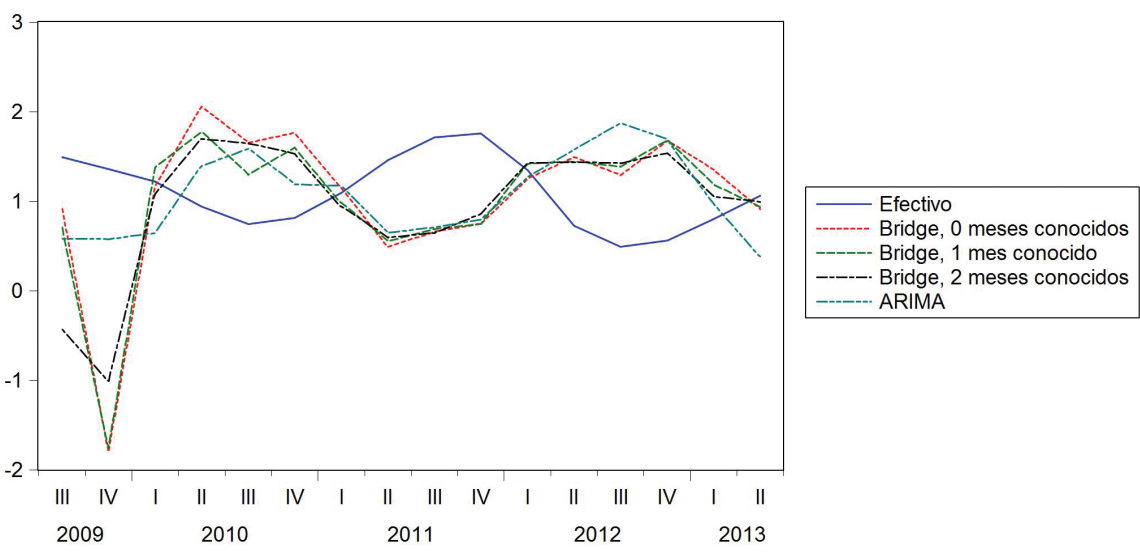

3 meses conocidos del trimestre vigente

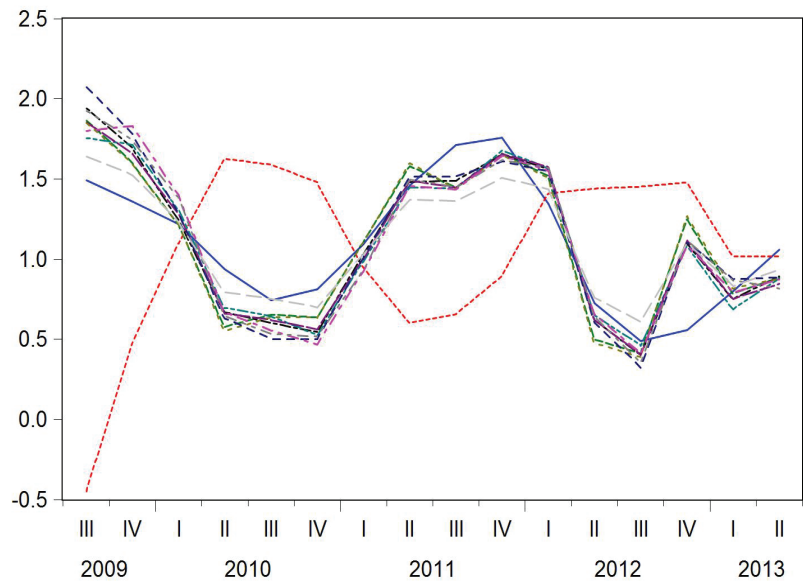

\begin{tabular}{|l|}
\hline - Efectivo \\
----- Bridge \\
----- MiDaS, 3 rezagos \\
---- MiDaS, 6 rezagos \\
---- MiDaS, 12 rezagos \\
---- MiDaS, AR(1), 3 rezagos \\
---- MiDaS, AR(1), 6 rezagos \\
---- MiDaS, AR(1), 9 rezagos \\
---- MiDaS, AR(1), 12 rezagos \\
--- Combinación \\
\hline
\end{tabular}

Fuente: Elaboración propia. 